\title{
Isogeometric analysis for numerical plate testing of dry woven fabrics involving frictional contact at meso-scale
}

\author{
S. Nishi ${ }^{1} \cdot$ K. Terada ${ }^{2} \cdot$ I. Temizer ${ }^{3}$ \\ Received: 4 June 2018 / Accepted: 11 December 2018 / Published online: 1 January 2019 \\ (c) Springer-Verlag GmbH Germany, part of Springer Nature 2019
}

\begin{abstract}
With a view to application to meso-macro decoupled two-scale draping simulations of dry woven fabrics, the method of isogeometric analysis (IGA) is applied to the numerical plate testing (NPT) for their periodic unit structures involving frictional contact at meso-scale. The meso-structure having periodicity only in in-plane directions is identified with a representative volume element to characterize the macroscopic mechanical behavior that reflects the interfacial frictional contact phenomenon between fiber bundles. NURBS basis functions are utilized to accurately solve macro-scale frictional contact problems and the mortar-based knot-to-surface algorithms are employed to evaluate the contact- and friction-related variables. A weaving process is simulated as a preliminary analysis to obtain the initial state of an in-plane unit cell that is subjected to bending of fiber bundles contacting with each other. Several numerical examples are presented to demonstrate the performance and capability of the proposed method of IGA-based NPT for characterizing the macroscopic structural responses of dry woven fabrics that can be substituted by macroscopic 'inelastic material' behaviors.
\end{abstract}

Keywords Isogeometric analysis $\cdot$ Frictional contact $\cdot$ Numerical plate testing $\cdot$ Dry woven fabric $\cdot$ Homogenization

\section{Introduction}

Owing to the superior performance while being light-weight, composite materials typified by fiber-reinforced plastics are widely used in various engineering fields such as aviation and automobile industries. At the same time, as various macroscopic material responses can be achieved according to the mechanical characteristics, distribution morphology and blend ratio of fiber and matrix materials, the concept of computer-aided engineering (CAE) has been introduced in the tailoring process of the macroscopic material properties by designing the micro-structures. The underlying idea

K. Terada

tei@irides.tohoku.ac.jp

S. Nishi

shinnosuke.nishi.p5@dc.tohoku.ac.jp

1 Department of Civil and Environmental Engineering, Tohoku University, Aza-Aoba 468-1, Aramaki, Aoba-ku, Sendai 980-8572, Japan

2 International Research Institute of Disaster Science, Tohoku University, Aza-Aoba 468-1, Aramaki, Aoba-ku, Sendai 980-8572, Japan

3 Department of Mechanical Engineering, Bilkent University, 06800 Ankara, Turkey to bridge between micro- and macro-structures goes back a long way and related methodologies are wide-ranging. But, in view of the characterization of various nonlinear behavior of composite materials within the CAE framework, the superiority of the computational homogenization, which is based on mathematical homogenization theory $[1,2]$, must be obvious [3,4]; see also some comprehensive review articles $[5,6]$. Despite the outstanding technological advances in the computational homogenization made during the 1990's and 2000's, considerable attention is still being paid to its extensive applications in practice. Among them, the micro-macro coupling scheme, which was first presented by Terada and Kikuchi $[7,8]$ and is often called $\mathrm{FE}^{2}$ method $[9,10]$, is still an important subject for study, as it does not require explicit function forms of macroscopic constitutive laws.

Although the micro-macro coupled computational homogenization is attractive because of its versatility for a variety of nonlinear problems, it seems unfortunately difficult to implement the method into existing general-purpose finite element (FE) programs and to take it to the market. To overcome the difficulty, Terada et al. [11] reconsidered the underlying theory [12] and proposed a micro-macro decoupling scheme, with which micro- and macro-scale analyses 
are separately conducted. In this method, a series of numerical analyses of a periodic micro-structure (unit cell), which is called numerical material testing (NMT), just provides numerical relationships between macroscopic stresses and strains under the assumption that a function form of the macroscopic constitutive equation can be determined with reference to the ones employed for the constituent materials at micro-scale. Then, by the identification of its material parameters with some optimization scheme, the homogenization procedure is completed. The localization analysis after macroscopic analyses is also possible within the limit of accuracy of the assumed macroscopic constitutive law. Although the decoupling method makes a sacrifice of versatility, nonlinear two-scale analyses can be realized with significantly low cost in comparison with the coupling scheme. For this reason, a cooperative system organized by the second author has successfully placed it into commercial use [13-17].

The decoupling method has recently been extended for composite plates, whose micro-structure have periodicity only in in-plane directions [18]. In this method, a series of microscopic analyses, called the numerical plate testing (NPT), is performed on the micro-structure having in-plane periodicity, called in-plane unit cell, to obtain the relationships between macroscopic resultant stresses and generalized strains; see also a similar approach in [19] in consideration of geometrically nonlinear effect on local buckling. The present study is for the application of the method of isogeometric analysis (IGA) to mesoscopic models, which are predominantly governed by contact behavior, so that it can be incorporated into the aforementioned micro-macro decoupling scheme. While nonlinear homogenization for composite plates and shells has been widely studied with a special interest in computational aspects $[20,21]$ and some peculiar mechanical behavior such as local buckling [22] and damage [23], most of the developments are presented within the $\mathrm{FE}^{2}$ framework and not intended to enhance mesoscopic analyses of composite plates and shells within the decoupling framework equipped with IGA.

The method of IGA proposed by Hughes [24] falls in the scope of FEM, but its numerical models have strict conformity with CAD models. Also, it enhances the existing FE methodology so as to accommodate spline functions such as NURBS for discretization of variables. Moreover, in IGA, an arbitrary order of continuity can be guaranteed at any point of the analysis domain and both mesh refinement and order elevation of spline functions are relatively easy tasks. Owing to these advantages, the method of IGA is applied to not only structural analyses with solid and shell elements $[25,26]$, but also various analyses in electromagnetic [27], damage/fracture [28] and structural optimization $[29,30]$ problems. In particular, since the higher-order continuity of spline functions can be of advantage in dealing with frictional contact problems, the method of IGA drew intense research interest in its performance for accuracy, computational efficiency and so on; see intensive investigations in [31-37]. In fact, since the shape representation of the boundary surface of elements is $C^{0}$-continuous in the standard FEM, the variation of a outward normal vector on the surface is discontinuous and therefore can cause convergence problems in the case of frictional sliding on it. This kind of difficulty can be overcome in IGA, because the smooth representation of the surface is possible. In addition, nonnegativity of spline basis functions is also advantageous for discretization of contact variables in an mortar-based setting.

In spite of these advantages, only few attempts have so far been made at computational homogenization along with the method of IGA to the best of the author's knowledge. The first trial in this context is carried out by Matsubara et al. [38], who discuss the points of attention in modeling multiple patches for multiple materials and in treating constraint conditions unique to NMT and NPT. However, it does not necessarily state their belief in the superiority of IGbased computational homogenization over FE-based one. The advantage of IGA-based surface representation was first utilized by Temizer [39] to establish the computational contact homogenization of soft matter friction involving micro-scale asperities. The effective use of IGA in microscale frictional contact problems is a good example of IG homogenization analysis (IGHA), but limited to the characterization of macroscopic friction behavior, and neither works on the volumetric homogenization nor reflects the interfacial frictional contact within a unit cell in the macroscopic friction coefficients.

With the above-mentioned background, this paper presents a method of IGA-based NPT for solving frictional contact problems in meso-structures of dry woven fabrics with a view to application to meso-macro decoupled two-scale draping simulations. Although computational homogenization of dry woven fabrics itself has formed one field of study, there are a variety of topics of key interest. For instance, Fillep et al. formulate the two-scale model of a dry woven fabric with finite deformation kinematics to conduct $\mathrm{FE}^{2}$ and database-based methods; see [40,41], respectively. Also, some researchers pay attention to the modeling and analysis of the mesostructures of dry woven fabrics, because the evaluation of damages of fibers in a bundle and fiber bundles subjected to frictional contact conditions must be an important issue in view of manufacturing process. To name a few, and [42] make a study on the determination of appropriate boundary conditions for a mesostructure, while Durville $[43,44]$ incorporate the effect of frictional contacts into their direct numerical simulations of a mesostructure of a dry woven fabric. Despite the considerations of frictional contact between adjacent fiber bundles, 
all these previous studies have not been enriched by the use of IGA.

In our approach, the virtual specimen in NPT is an inplane unit cell composed of woven fiber bundles only, which contact with each other, and is identified with a representative volume element (RVE) to characterize the macroscopic mechanical behavior that reflects the interfacial frictional contact phenomenon between fiber bundles. If we consider a matrix part together with fiber bundles, some special techniques such as hierarchical splines and adaptive remeshing are needed [45] because of its complicated interface conditions. Also, to improve the accuracy in solving frictional contact between fiber bundles, it has been confirmed that adaptive remeshing techniques would be effect $[46,47]$. Nonetheless, taking different views from NPTs, we confine ourselves solely to the use of the standard NURBS basis functions in the IG discretization for solving meso-scale frictional contact problems defined in an in-plane unit cell to characterize the macroscopic responses of dry woven fabrics. The penalty method and the mortarbased knot-to-surface (KTS) [32-34] algorithm, which avoids the locking and over-constraining caused by the penalty parameter, are employed to satisfy the contact constraints. We also discuss the use of an in-plane subunit cell, which is a one quarter of an in-plane unit cell, along with special constraint conditions to stabilize NPT analyses. Furthermore, virtual in-plane sub-unit cells are introduced to deal with in case of in-plane shear deformations, since contact surfaces are possibly outside the analysis domain.

Several numerical examples are presented to demonstrate the performance and capability of the proposed method of IGA-based NPT for characterizing the macroscopic structural responses of dry woven fabrics that can be substituted by macroscopic 'inelastic material' behaviors. After a simple numerical test for computational efficiency is conducted to demonstrate the performance of the IGA with the NURBS basis functions in solving frictional contact problems for fiber-bundles during NPT, a weaving process is simulated as a preliminary analysis to obtain the initial state of an in-plane unit cell that is subjected to bending of fiber bundles contacting with each other. Then, we exemplify the IGA-based NPTs by characterizing the macroscopic in-plane and out-of-plane mechanical behaviors of dry woven fabrics with a view to application to decoupled two-scale analyses. In particular, in-plane shear deformations of dry woven fabrics, which are subjected to pre-tensioning stress [48,49], is known to exhibit dependency on the friction behavior between fiber bundles and is subject to discussions as to devising an appropriate macroscopic analysis model composed of 'inelastic' materials.

\section{Computational homogenization for composite plates}

With a view to characterization of macroscopic mechanical behavior of dry woven fabrics, we summarize the method of numerical plate testing (NPT) for general thin composite plates within the framework of finite strain theory, which is just an extension of that formulated within the small strain framework [18]. While the macrostructure is supposed to be a plate or shell, the mesostructure is regarded as a solid and is assumed to be periodically arranged only in the in-plane directions at a macro-scale. The minimum periodic unit is then regarded as a representative volume element (RVE) for the characterization of the macroscopic mechanical behavior and is referred to as an in-plane unit cell (UC) in this study.

Figure 1 shows the in-plane UC of a typical dry woven fabric whose kinematics is described by the mesoscopic coordinate system $\boldsymbol{Y}$. This in-plane UC is assumed to be located at a macroscopic material point and identified with position vector $\boldsymbol{X}$ in the initial configuration. Let $\boldsymbol{x}=\tilde{\phi}(\boldsymbol{X})$ define the motion of this material point and be a position vector in the current configuration. Then, the macroscopic deformation gradient is defined as $\tilde{\boldsymbol{F}}=\nabla_{X} \tilde{\boldsymbol{\phi}}(\boldsymbol{X})=\tilde{\boldsymbol{H}}+\mathbf{1}$, where $\tilde{\boldsymbol{H}}$ is the macroscopic displacement gradient and $\mathbf{1}$ is the second-order identity tensor.

According to the method of linear NPT presented in [18], the mesoscopic displacement is defined as

$$
\boldsymbol{w}=\left[\begin{array}{ccc}
\tilde{H}_{1}+Z_{3} \tilde{H}_{4} & \frac{1}{2}\left(\tilde{H}_{3}+Z_{3} \tilde{H}_{6}\right)-\frac{1}{2} Z_{2} \tilde{H}_{6} \\
\frac{1}{2}\left(\tilde{H}_{3}+Z_{3} \tilde{H}_{6}\right) & \tilde{H}_{2}+Z_{3} \tilde{H}_{5} & -\frac{1}{2} Z_{1} \tilde{H}_{6} \\
-\frac{1}{2} Z_{2} \tilde{H}_{6} & -\frac{1}{2} Z_{1} \tilde{H}_{6} & 0
\end{array}\right] \boldsymbol{Y}+\boldsymbol{u}^{*},
$$

in which the effects of transverse shear deformation are not reflected. Here, $\tilde{H}_{1}$ and $\tilde{H}_{2}$ represent the macroscopic inplane normal deformations in the $Y_{1}$ and $Y_{2}$ directions (Mode

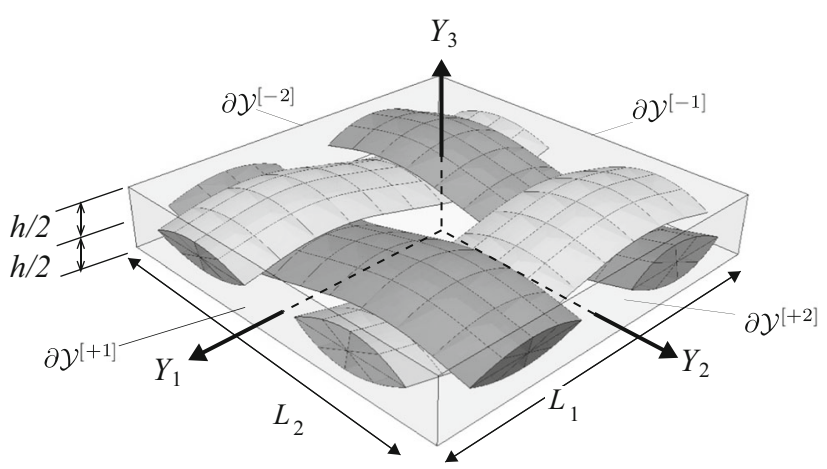

Fig. 1 In-plane unit cell 


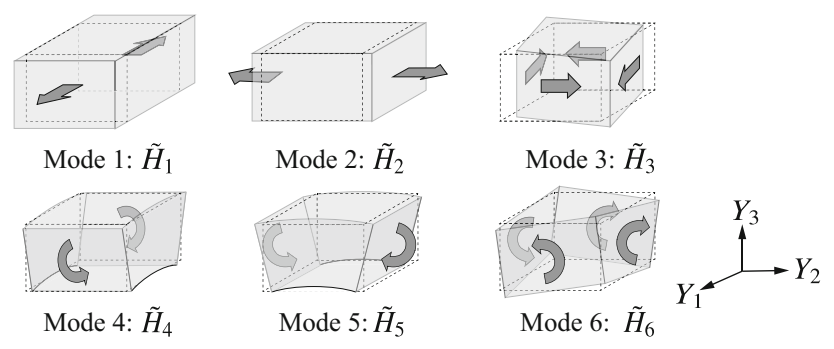

Fig. 2 Deformation modes of macroscopic generalised displacement gradient

1 and 2), and $\tilde{H}_{3}$ is the macroscopic in-plane shear deformation (Mode 3 ). Also, $\tilde{H}_{4}$ and $\tilde{H}_{5}$ represent the macroscopic bending deformations about axes $Y_{1}$ and $Y_{2}$ (Mode 4 and 5), and $\tilde{H}_{6}$ the macroscopic torsional deformation (Mode 6). All these components are constant with respect to the mesoscopic coordinate $\boldsymbol{Y}$ and the corresponding deformation modes are depicted in Fig. 2. In this study, we call $\tilde{H}_{i}(i=1, \ldots, 6)$ the macroscopic generalized displacement gradients for convenience, although they might not be consistent with the proper description of macroscopic kinematics. In addition, we have introduced an additional mesoscopic coordinate system $\boldsymbol{Z}$, which is independent of $\boldsymbol{Y}$, to control the mesoscopic deformations with the macroscopic generalized displacement gradients, along the line of the linear NPT presented in Reference [18]. Here, this $Z_{i}$ are set as cellcentered axes and axis $Z_{3}$ in the thickness direction has the same measure as axis $X_{3}$ at the macroscale. Furthermore, $\boldsymbol{u}^{*}$ is the so-called fluctuation displacement vector that satisfies the following in-plane periodicity conditions:

$\left.\boldsymbol{u}^{*}\right|_{\partial y_{0}^{[+J]}}=\left.\boldsymbol{u}^{*}\right|_{\partial y_{0}^{[-J]}} \quad(J=1,2)$,

where $\partial \mathcal{Y}_{0}^{[+J]}$ and $\partial \mathcal{Y}_{0}^{[-J]}$ are the opposing boundary surfaces in the initial configuration, which are assumed to be in parallel. Here, $J$ taking 1 and 2 indicates the boundary surfaces associated with $Y_{1^{-}}$and $Y_{2}$-planes, respectively.

Then, the substitution of Eq. (1) into (2) yields the following constraint conditions:

$\left\{\begin{array}{l}w_{1}^{[1]}-w_{1}^{[-1]}=\left(\tilde{H}_{1}+Z_{3} \tilde{H}_{4}\right) L_{1} \\ w_{2}^{[1]}-w_{2}^{[-1]}=\frac{1}{2}\left(\tilde{H}_{3}+Z_{3} \tilde{H}_{6}\right) L_{1} \\ w_{3}^{[1]}-w_{3}^{[-1]}=-\frac{1}{2} Z_{2} \tilde{H}_{6} L_{1}\end{array}\right.$,

$$
\left\{\begin{array}{l}
w_{1}^{[2]}-w_{1}^{[-2]}=\frac{1}{2}\left(\tilde{H}_{3}+Z_{3} \tilde{H}_{6}\right) L_{2} \\
w_{2}^{[2]}-w_{2}^{[-2]}=\left(\tilde{H}_{2}+Z_{3} \tilde{H}_{5}\right) L_{2} \\
w_{3}^{[2]}-w_{3}^{[-2]}=-\frac{1}{2} Z_{1} \tilde{H}_{6} L_{2}
\end{array} .\right.
$$

Here, $w^{[J]}$ is the displacement on the boundary surface $\partial \mathcal{Y}^{[J]}$, and $L_{J}(J=1,2)$ are the widths of the in-plane $\mathrm{UC}$ as shown in Fig. 1.

The derivative of the mesoscopic displacement (1) with respect to $\boldsymbol{Y}$ derives the 'mesoscopic' deformation gradient as

$$
\begin{aligned}
\boldsymbol{F}= & {\left[\begin{array}{ccc}
\tilde{H}_{1} & \frac{1}{2} \tilde{H}_{3} & -\frac{1}{2} Z_{2} \tilde{H}_{6} \\
\frac{1}{2} \tilde{H}_{3} & \tilde{H}_{2} & -\frac{1}{2} Z_{1} \tilde{H}_{6} \\
-\frac{1}{2} Z_{2} \tilde{H}_{6} & -\frac{1}{2} Z_{1} \tilde{H}_{6} & 0
\end{array}\right] } \\
& +Z_{3}\left[\begin{array}{ccc}
\tilde{H}_{4} & \frac{1}{2} \tilde{H}_{6} & 0 \\
\frac{1}{2} \tilde{H}_{6} & \tilde{H}_{5} & 0 \\
0 & 0 & 0
\end{array}\right]+\nabla_{Y} \boldsymbol{u}^{*}+\mathbf{1}
\end{aligned}
$$

With this mesoscopic deformation gradient being input data, the mesoscopic first Piola-Kirchhoff stress $\boldsymbol{P}$ is determined by an arbitrary constitutive model so as to satisfy the following mesoscopic equilibrium equation:

$\nabla_{Y} \cdot \boldsymbol{P}=\mathbf{0}$

After the equilibrated stress is obtained, with reference to the in-plane homogenization formula [18], the mesoscopic first Piola-Kirchhoff stress $\boldsymbol{P}$ can be related to the macroscopic first Piola-Kirchoff resultant stresses (hereinafter, these are referred to as "Macro-PK1-RS"), respectively, as

$$
\begin{aligned}
& \tilde{\boldsymbol{N}}=\int_{-h / 2}^{h / 2}\left[\frac{1}{L_{1} L_{2}} \int_{-L_{1} / 2}^{L_{1} / 2} \int_{-L_{2} / 2}^{L_{2} / 2} \boldsymbol{P} d Y_{1} d Y_{2}\right] d Z_{3}, \\
& \tilde{\boldsymbol{M}}=\int_{-h / 2}^{h / 2} Z_{3}\left[\frac{1}{L_{1} L_{2}} \int_{-L_{1} / 2}^{L_{1} / 2} \int_{-L_{2} / 2}^{L_{2} / 2} \boldsymbol{P} d Y_{1} d Y_{2}\right] d Z_{3},
\end{aligned}
$$

where $h$ is the height of the in-plane UC as shown in Fig. 1.

\section{Frictional contact problem}

A frictional contact problem for two bodies and its numerical treatment with the penalty method are outlined by reference to the formulation in [50].

\subsection{Contact and friction conditions}

Let us consider the two bodies $\mathcal{B}^{\gamma}(\gamma=1,2)$ contacting with each other in the current configuration, as shown in Fig. 3, in 


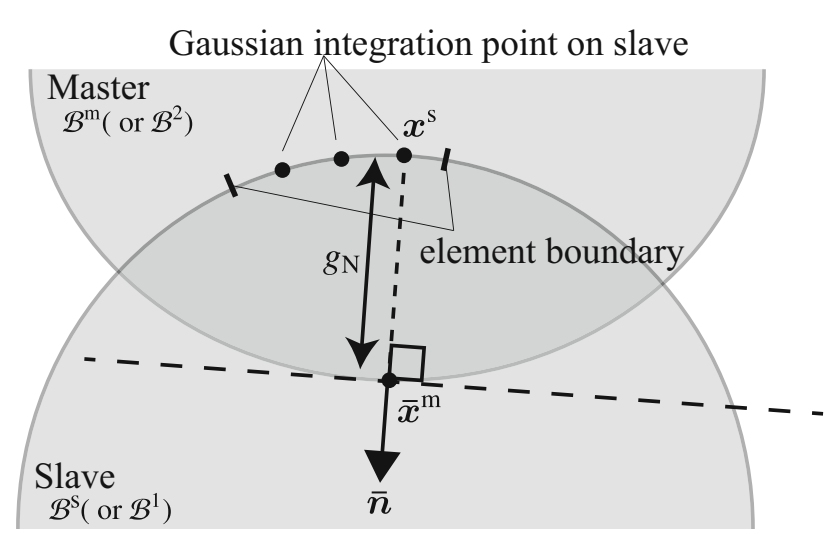

Fig. 3 Illustration of normal gap displacement $g_{\mathrm{N}}$

which the lower and upper domains are indicated by "Slave" and "Master". Also, let $\boldsymbol{x}^{\mathrm{s}}$ and $\boldsymbol{x}^{\mathrm{m}}$ be position vectors on the boundary surfaces of Slave and Master in the current configurations, respectively. Then, the distance between these points, which is referred to as the gap, can be defined as

$g_{\mathrm{N}}:=\left(\boldsymbol{x}^{\mathrm{s}}-\overline{\boldsymbol{x}}^{\mathrm{m}}\right) \cdot \overline{\boldsymbol{n}}$

Here, $\overline{\boldsymbol{x}}^{\mathrm{m}}:=\boldsymbol{x}^{\mathrm{m}}(\overline{\boldsymbol{\xi}})$ is the position vectors of a point on the boundary surface of Master that minimizes the distance $d(\boldsymbol{\xi}):=\left\|\boldsymbol{x}^{\mathrm{s}}-\overline{\boldsymbol{x}}^{\mathrm{m}}(\boldsymbol{\xi})\right\|$ relative to the Gaussian integration point $\boldsymbol{x}^{\mathrm{s}}$ on the boundary surface of Slave, and $\overline{\boldsymbol{n}}=\boldsymbol{n}(\overline{\boldsymbol{\xi}})$ is the outward unit normal vector on the point. Also, $\xi$ is the position vector measured in the two-dimensional (2D) natural coordinate system, which will be introduced in the geometric representation for IGA later, and is related to the position vector $\boldsymbol{x}(\boldsymbol{\xi})$ in the physical coordinate system through the parametric mapping. Therefore, to determine $\overline{\boldsymbol{x}}^{\mathrm{m}}$ in Eq. (9), the following equation must be solved for the position vector $\bar{\xi}=\left(\bar{\xi}^{1}, \bar{\xi}^{2}\right)$ :

$\left(\boldsymbol{x}^{\mathrm{s}}-\overline{\boldsymbol{x}}^{\mathrm{m}}\right) \cdot \overline{\boldsymbol{a}}_{\alpha}=0(\alpha=1,2)$,

where $\overline{\boldsymbol{a}}_{\alpha}=\boldsymbol{x}_{, \alpha}^{\mathrm{m}}(\overline{\boldsymbol{\xi}})=\mathrm{d} \boldsymbol{x}^{\mathrm{m}} / \mathrm{d} \xi^{\alpha}(\overline{\boldsymbol{\xi}})$ is the first derivative of $\boldsymbol{x}^{\mathrm{m}}$ with respect to $\boldsymbol{\xi}$ at point $\bar{\xi}$ on the boundary surface of Master, and represents to the tangential plane at this point. Since the parametric mapping $\boldsymbol{x}(\boldsymbol{\xi})$ is a nonlinear function of $\xi$ in the geometric representation with NURBS basis functions, the nonlinear solution methods such as the Newton-Raphson method have to be employed to solve it for $\bar{\xi}$.

In a non-contact situation, the contact pressure $p_{\mathrm{N}}$ is zero and the normal gap $g_{\mathrm{N}}$ is positive. On the other hand, in case of contact, the contact pressure $p_{\mathrm{N}}$ must be positive, while the normal gap $g_{\mathrm{N}}$ must be zero, as the two bodies cannot penetrate into each other. Thus, the following Karush-KuhnTucker (KKT) conditions have to be satisfied:
$g_{\mathrm{N}} \geq 0, \quad p_{\mathrm{N}} \leq 0, \quad g_{\mathrm{N}} p_{\mathrm{N}}=0$

When $p_{\mathrm{N}}<0$ is satisfied, i.e., the two bodies contact with each other, the following Coulomb friction law is employed in this study:

$f_{\mathrm{s}}=\left\|\boldsymbol{t}_{\mathrm{T}}\right\|-\mu_{\text {fric }}\left|p_{\mathrm{N}}\right| \leq 0$,

where $\mu_{\text {fric }}$ is the friction coefficient. Here, $t_{\mathrm{T}}$ is the traction vector in the tangential direction, along which the slip rate vector is assumed to satisfy the following flow rule:

$\dot{\boldsymbol{g}}_{\mathrm{T}}^{\text {slip }}=\dot{\gamma} \frac{\boldsymbol{t}_{\mathrm{T}}}{\left\|\boldsymbol{t}_{\mathrm{T}}\right\|}$

where $\dot{\gamma} \geq 0$ is the magnitude of the slip vector. Here, $\boldsymbol{g}_{\mathrm{T}}^{\text {slip }}$ is the displacement vector of the contact point that evolves only when slip condition is satisfied, i.e., $f_{\mathrm{s}}=0$ in Eq. (12), and its time rate of change follows the flow rule (13). The combination of these inequalities along with the complementary condition constitute the following KKT conditions as

$\dot{\gamma} \geq 0, \quad f_{\mathrm{s}} \leq 0, \quad \dot{\gamma} f_{\mathrm{s}}=0$.

\subsection{Virtual work for frictional contact}

Let us consider the equilibrated state of two bodies contacting with each other and denote the internal and surface domains in the initial configuration by $\mathcal{B}_{0}^{\gamma}$ and $\Gamma_{0}^{\gamma}$, respectively. Then, the equilibrium equation takes the following weak form:

$\sum_{\gamma}^{2}\left\{\int_{\mathcal{B}_{0}^{\gamma}} \delta \boldsymbol{F}: \boldsymbol{P} \mathrm{d} V-\int_{\Gamma_{0}^{\gamma}} \delta \boldsymbol{w} \cdot \overline{\boldsymbol{T}} \mathrm{d} A\right\}-C_{\mathrm{c}}=0$

where $\overline{\boldsymbol{T}}$ is the given traction vector on the Neumann boundary $\Gamma_{0}^{\gamma}$ in the initial configuration. Here, the body force is neglected. Also, $\delta \boldsymbol{w}$ is the virtual displacement, by which the variation of the deformation gradient $\delta \boldsymbol{F}$ is defined. The term $C_{\mathrm{c}}$ associated with the frictional contact between these bodies is defined as

$C_{\mathrm{c}}=\int_{\Gamma_{\mathrm{c} 0}}\left(\delta g_{\mathrm{N}} p_{\mathrm{N}}+\delta \boldsymbol{g}_{\mathrm{T}} \cdot \boldsymbol{t}_{\mathrm{T}}\right) \mathrm{d} A$,

where $\Gamma_{\mathrm{c} 0}$ is the surface domain pulled back to the initial configuration from $\Gamma_{\mathrm{c}}$ in the current configuration, on which the contact condition $g_{\mathrm{N}} \leq 0$ is satisfied. Also, the variation of $g_{\mathrm{N}}$ is given as

$\delta g_{\mathrm{N}}=\left(\delta \boldsymbol{w}^{\mathrm{s}}-\delta \overline{\boldsymbol{w}}^{\mathrm{m}}\right) \cdot \overline{\boldsymbol{n}}$

in which the relationship condition $\boldsymbol{x}^{\mathrm{s}}-\overline{\boldsymbol{x}}^{\mathrm{m}}=\delta g_{\mathrm{N}} \overline{\boldsymbol{n}}$ has been reflected so that $\left(\boldsymbol{x}^{\mathrm{s}}-\overline{\boldsymbol{x}}^{\mathrm{m}}\right) \cdot \delta \overline{\boldsymbol{n}}=\delta g_{\mathrm{N}} \overline{\boldsymbol{n}} \cdot \delta \overline{\boldsymbol{n}}=0$. 
On the other hand, the total displacement in the tangential direction is denoted by $\boldsymbol{g}_{\mathrm{T}}$ and its time rate of change and the variation are respectively given as

$$
\begin{aligned}
\dot{\boldsymbol{g}_{\mathrm{T}}} & =\dot{\xi}^{\alpha} \overline{\boldsymbol{a}}_{\alpha}, \\
\delta \boldsymbol{g}_{\mathrm{T}} & =\delta \xi^{\alpha} \overline{\boldsymbol{a}}_{\alpha} .
\end{aligned}
$$

Here, the variation $\delta \xi^{\alpha}$ of $\xi^{\alpha}$ can be derived by taking variation of the relationship (10) such that

$\delta \xi^{\alpha}=\bar{H}^{\alpha \beta}\left[\left(\delta \boldsymbol{w}^{\mathrm{s}}-\delta \overline{\boldsymbol{w}}^{\mathrm{m}}\right) \cdot \boldsymbol{a}_{\alpha}+g_{\mathrm{N}} \overline{\boldsymbol{n}} \cdot \delta \overline{\boldsymbol{w}}_{, \alpha}^{\mathrm{m}}\right]$,

where $\bar{H}^{\alpha \beta}$ is the inverse matrix of

$\bar{H}_{\alpha \beta}=\bar{a}_{\alpha \beta}+g_{\mathrm{N}} \overline{\boldsymbol{n}} \cdot \overline{\boldsymbol{x}}_{, \alpha \beta}^{\mathrm{m}}$.

Here, $\bar{a}_{\alpha \beta}:=\overline{\boldsymbol{a}}_{\alpha} \cdot \overline{\boldsymbol{a}}_{\beta}$ is the covariant metric matrix and $\overline{\boldsymbol{x}}_{, \alpha \beta}^{\mathrm{m}}:=\partial^{2} \overline{\boldsymbol{x}}^{\mathrm{m}} / \partial \xi \partial \xi^{\mathrm{fi}}$ is the second derivative of $\overline{\boldsymbol{x}}^{\mathrm{m}}$ with respect to $\xi$. With the contravariant vector $\overline{\boldsymbol{a}}^{\alpha}$ corresponding to $\overline{\boldsymbol{a}}_{\alpha}$, the traction vector in the tangential direction can be represented as $\boldsymbol{t}_{\mathrm{T}}=t_{\mathrm{T} \alpha} \overline{\boldsymbol{a}}^{\alpha}$. Therefore, the weak form yields

$C_{\mathrm{c}}=\int_{\Gamma_{\mathrm{c} 0}}\left(\delta g_{\mathrm{N}} p_{\mathrm{N}}+\delta \xi^{\alpha} t_{\mathrm{T} \alpha}\right) \mathrm{d} A$,

in which Eq. (19) has been utilized. With the help of these expressions, the linearized version of Eq. (15) can be derived for the Newton-Raphson's iterative procedure; see [50] in detail.

\subsection{Penalty method}

In the penalty method, the contact pressure $p_{\mathrm{N}}$ in Eq. (16), which is associated with the contact in the normal direction, is approximated as

$p_{\mathrm{N}}=\epsilon_{\mathrm{N}} \bar{g}_{\mathrm{N}}, \quad \bar{g}_{\mathrm{N}}=\left\{\begin{array}{ll}g_{\mathrm{N}} & \text { if } g_{\mathrm{N}}<0 \\ 0 & \text { otherwise. }\end{array}\right.$,

where $\epsilon_{\mathrm{N}}$ is the penalty parameter for the normal contact.

The variables in the above equations for the tangential slip can be calculated by the algorithm described below. In the description, time rates of change are approximated by the backward difference scheme; e.g., $\xi^{\alpha}=\left(\xi_{n+1}^{\alpha}-\xi_{n}^{\alpha}\right) / \Delta t$ where $\Delta t$ is the time increment and subscripts, $n+1$ and $n$, indicate the current and previous time steps, respectively.

Suppose that the displacement field has been determined during the Newton-Raphson iterative process for time step $n+1$. Then, we introduce the predictor of $t_{\mathrm{T} \alpha}$ at time step $n+1$ as
$t_{\mathrm{T}, n+1}^{\text {trial }}=t_{\mathrm{T}, n}+\epsilon_{\mathrm{T}} a_{\alpha \beta}\left(\xi_{n+1}^{\beta}-\xi_{n}^{\beta}\right)$.

where $\epsilon_{\mathrm{T}}$ is the penalty parameter for the tangential contact. When the condition $f_{\mathrm{s}, n+1}^{\text {trial }}=\left\|\boldsymbol{t}_{\mathrm{T}, n+1}^{\text {trial }}\right\|-\mu_{\text {fric }}\left|p_{\mathrm{N}, n+1}\right|>0$ is met, the traction vector in the tangential direction becomes

$$
\begin{aligned}
\boldsymbol{t}_{\mathrm{T}, n+1} & =\boldsymbol{t}_{\mathrm{T}, n}+\epsilon_{\mathrm{T}} \Delta t\left(\dot{\boldsymbol{g}}_{\mathrm{T}, n+1}-\dot{\boldsymbol{g}}_{\mathrm{T}, n+1}^{\text {slip }}\right) \\
& =\boldsymbol{t}_{\mathrm{T}, n+1}^{\mathrm{trial}}-\epsilon_{\mathrm{T}} \Delta t \dot{\boldsymbol{g}}_{\mathrm{T}, n+1}^{\text {slip }} \\
& =\boldsymbol{t}_{\mathrm{T}, n+1}^{\mathrm{trial}}-\epsilon_{\mathrm{T}} \Delta \gamma \frac{\boldsymbol{t}_{\mathrm{T}, n+1}^{\mathrm{trial}}}{\left\|\boldsymbol{t}_{\mathrm{T}, n+1}^{\mathrm{trial}}\right\|},
\end{aligned}
$$

where the flow rule (13) has been utilized and $\Delta \gamma=\dot{\gamma} \Delta t$ is the increment of slip displacement. By multiplying the both sides of this equation by $\boldsymbol{t}_{\mathrm{T}, n+1}^{\text {trial }} /\left\|\boldsymbol{t}_{\mathrm{T}, n+1}^{\text {trial }}\right\|$, we obtain the norm of the traction vector as $\left\|\boldsymbol{t}_{\mathrm{T}, n+1}\right\|=\left\|\boldsymbol{t}_{\mathrm{T}, n+1}^{\text {trial }}\right\|-\epsilon_{\mathrm{T}} \Delta \gamma$, by which $\Delta \gamma$ that satisfies $f_{\mathrm{s}, n+1}=0$ yields

$\Delta \gamma=\frac{1}{\epsilon_{\mathrm{T}}}\left(\left\|\boldsymbol{t}_{\mathrm{T}, n+1}^{\mathrm{trial}}\right\|-\mu_{\text {fric }}\left|p_{\mathrm{N}, n+1}\right|\right)$.

Therefore, $t_{\mathrm{T} \alpha}$ can be calculated as

$t_{\mathrm{T}}=\left\{\begin{array}{lll}t_{\mathrm{T}, n+1}^{\text {trial }} & \text { if } & f_{\mathrm{s}, n+1}^{\text {trial }} \leq 0 \\ -\mu_{\text {fric }}\left|p_{\mathrm{N}, n+1}\right| \frac{t_{\mathrm{T}, n+1}^{\text {trial }}}{\left\|\boldsymbol{t}_{\mathrm{T}, n+1}^{\text {trial }}\right\|} & \text { if } & f_{\mathrm{s}, n+1}^{\text {trial }}>0\end{array}\right.$.

Here, the second equation has been obtained by substituting Eq. (26) into Eq. (25).

\section{Isogeometric analysis}

The method of isogeometric analysis (IGA) [24] with NURBS basis functions is summarized in this section. Although the ways of approximation in IGA and standard FEM are different, little difference is seen in their discretization procedures. Therefore, we just outline the method of discretization with the NURBS basis functions along with their characteristics.

\subsection{Geometric representation with NURBS functions}

Non-Uniformed Rational B-Spline (NURBS) functions basis functions in a three-dimensional (3D) space can be generated from B-spline functions $N^{1}\left(\xi^{1}\right), N^{2}\left(\xi^{2}\right)$ and $N^{3}\left(\xi^{3}\right)$ defined with three separate knot vectors $\boldsymbol{\Xi}^{1}, \boldsymbol{\Xi}^{2}$ and $\boldsymbol{\Xi}^{3}$ as

$$
R_{i, j, k}\left(\xi^{1}, \xi^{2}, \xi^{3}\right)=\frac{N_{i, p}^{1}\left(\xi^{1}\right) N_{j, q}^{2}\left(\xi^{2}\right) N_{k, r}^{3}\left(\xi^{3}\right) w_{i, j, k}}{W\left(\xi^{1}, \xi^{2}, \xi^{3}\right)},
$$


where we have defined

$W\left(\xi^{1}, \xi^{2}, \xi^{3}\right)=\sum_{i=1}^{n_{\mathrm{c}}} \sum_{j=1}^{m_{\mathrm{c}}} \sum_{k=1}^{l_{\mathrm{c}}} N_{i, p}^{1}\left(\xi^{1}\right) N_{j, q}^{2}\left(\xi^{2}\right) N_{k, r}^{3}\left(\xi^{3}\right) w_{i, j, k}$.

Here, B-spline basis functions $N_{i, p}^{a}\left(\xi^{a}\right)(a=1,2,3)$ can be calculated with the following Cox-de Boor's recursive formula:

$N_{i, p}^{a}(\xi)=\frac{\xi^{a}-\xi_{i}^{a}}{\xi_{i+p}^{a}-\xi_{i}^{a}} N_{i, p-1}^{a}\left(\xi^{a}\right)+\frac{\xi_{i+p+1}^{a}-\xi^{a}}{\xi_{i+p+1}^{a}-\xi_{i+1}^{a}} N_{i+1, p-1}^{a}\left(\xi^{a}\right)$.

$N_{i, 0}^{a}(\xi)= \begin{cases}1 & \text { if } \xi_{i}^{a} \leq \xi^{a} \leq \xi_{i+1}^{a} \\ 0 & \text { otherwise. }\end{cases}$

By introducing a knot vector $\boldsymbol{\Xi}^{a}=\left(\xi_{1}^{a}, \xi_{2}^{a}, \ldots, \xi_{n+p+1}^{a}\right)$, whose components $\xi_{i}^{a}$ are indices $i$ in Eqs. (30) and (31) and substituting them from the left to the right, we define a set of B-spline basis functions $N_{i, p}^{a}\left(\xi^{a}\right)$. With NURBS functions (28), the displacement vector $\boldsymbol{w}$ in the 3D space at a control point, which is identified with indices $i, j$ and $k$, is approximated as

$\boldsymbol{w}^{h}\left(\xi^{1}, \xi^{2}, \xi^{3}\right)=\sum_{i=1}^{n_{\mathrm{c}}} \sum_{j=1}^{m_{\mathrm{c}}} \sum_{k=1}^{l_{\mathrm{c}}} R_{i, j, k}\left(\xi^{1}, \xi^{2}, \xi^{3}\right) \hat{\boldsymbol{w}}_{i, j, k}$,

where $\hat{\boldsymbol{w}}_{i, j, k}$ are the displacement vectors of the corresponding control points.

The characteristic features of the basis functions used for both geometric representation and approximation of variables are listed as follows:

- possessing the partition-of-unity property,

- always non-negative, and

- $C^{p-1}$-continuous in its domain and on the element boundary when the order of NURBS functions is $p$,

in which the second and the third features are advantageous especially to frictional contact problems. In IGA, the discretization of variables such as displacements are approximated with the same set of basis functions $R_{i, j, k}\left(\xi^{1}, \xi^{2}, \xi^{3}\right)$ in Eq. (32).

\subsection{Discretization for frictional contact behavior}

To consider a boundary surface of a 3D body, the corresponding component of the knot vector is fixed at, e.g., $\xi^{3}=\xi_{0}^{3}$ with $k=k_{0}$ in Eq. (28), such that

$$
\boldsymbol{S}\left(\xi^{1}, \xi^{2}\right)=\sum_{i=1}^{n_{\mathrm{c}}} \sum_{j=1}^{m_{\mathrm{c}}} R_{i, j, k_{0}}\left(\xi^{1}, \xi^{2}\right) \boldsymbol{B}_{i, j, k_{0}}=\sum_{I=1}^{n_{\mathrm{c}}} R^{\mathrm{I}} \boldsymbol{B}^{\mathrm{I}}
$$

In the second equation here, notational simplification is introduced by indexing $I$ with the set $\left(i, j, k_{0}\right)$. Also, to distinguish the basis functions of the boundary surfaces on Slave and Master, respectively denoted by $R^{\mathrm{s}, \mathrm{I}}$ and $R^{\mathrm{m}, \mathrm{I}}$, the position vectors on them are expressed as $\boldsymbol{x}^{\mathrm{s}}=\sum_{I=1}^{n_{\mathrm{c}}} R^{\mathrm{s}, \mathrm{I}} \hat{\boldsymbol{x}}^{\mathrm{S}, \mathrm{I}}$ and $\overline{\boldsymbol{x}}^{\mathrm{m}}=\sum_{I=1}^{n_{\mathrm{c}}} R^{\mathrm{m}, \mathrm{I}} \hat{\boldsymbol{x}}^{\mathrm{m}, \mathrm{I}}$, respectively. Here, $\hat{\bullet}$ indicates the value at a control point. Using these expressions, Eqn. (9), (17) and (20) can be discretized as

$$
\begin{aligned}
g_{\mathrm{N}}= & \left(\sum_{I=1}^{n} R^{\mathrm{s}, \mathrm{I}} \hat{\boldsymbol{x}}^{\mathrm{s}, \mathrm{I}}-\sum_{I=1}^{n} R^{\mathrm{m}, \mathrm{I}} \hat{\boldsymbol{x}}^{\mathrm{m}, \mathrm{I}}\right) \cdot \overline{\boldsymbol{n}} \\
\delta g_{\mathrm{N}}= & \left(\sum_{I=1}^{n} R^{\mathrm{s}, \mathrm{I}} \delta \hat{\boldsymbol{w}}^{\mathrm{s}, \mathrm{I}}-\sum_{I=1}^{n} \bar{R}^{\mathrm{m}, \mathrm{I}} \delta \hat{\boldsymbol{w}}^{\mathrm{m}, \mathrm{I}}\right) \cdot \overline{\boldsymbol{n}}, \\
\delta \xi^{\alpha}= & \bar{H}^{\alpha \beta}\left[\left(\sum_{I=1}^{n} R^{\mathrm{s}, \mathrm{I}} \delta \hat{\boldsymbol{w}}^{\mathrm{s}, \mathrm{I}}-\sum_{I=1}^{n} R^{\mathrm{m}, \mathrm{I}} \delta \hat{\boldsymbol{w}}^{\mathrm{m}, \mathrm{I}}\right) \cdot \boldsymbol{a}_{\alpha}\right. \\
& \left.+g_{\mathrm{N}} \overline{\boldsymbol{n}} \cdot \sum_{I=1}^{n} \bar{R}_{, \alpha}^{\mathrm{m}, \mathrm{I}} \delta \hat{\boldsymbol{w}}^{\mathrm{m}, \mathrm{I}}\right] .
\end{aligned}
$$

It is known that the contact pressure sometimes oscillates due to overly strong constraints imposed on the contact surfaces, when a relatively large value is set for the penalty parameter [32]. To prevent this kind of trouble, the mortar-based formulation, which is presented in [32-34], is employed in this study. The idea of this method is to relax the constraints in the contact condition by evaluating the amount of penetration with the average value of the gaps at all the Gaussian integration points in an element. To be more specific, defining the areal mean of variable $*$ at a Gaussian integration point by $<*>=\int_{\Gamma_{\mathrm{c} 0}} * \mathrm{~d} A$, we respectively re-define the gap and tangential displacement at a control point $I$ as

$$
\begin{aligned}
g_{\mathrm{N}}^{\mathrm{I}} & =\frac{\left\langle g_{\mathrm{N}} R^{\mathrm{I}}\right\rangle}{\left\langle R^{\mathrm{I}}\right\rangle}, \\
\xi_{n+1}^{\alpha, \mathrm{I}} & =\frac{\left\langle\xi_{n+1}^{\alpha} R^{\mathrm{I}}\right\rangle}{\left\langle R^{\mathrm{I}}\right\rangle} .
\end{aligned}
$$

The states of contact and non-contact are judged by this value and the contact pressure at a control point is computed as follows:

$p_{\mathrm{N}}^{\mathrm{I}}=\epsilon_{\mathrm{N}} \bar{g}_{\mathrm{N}}^{\mathrm{I}} \quad \bar{g}_{\mathrm{N}}^{\mathrm{I}}=\left\{\begin{array}{ll}g_{\mathrm{N}}^{\mathrm{I}} & \text { if } g_{\mathrm{N}}^{\mathrm{I}} \leq 0 \\ 0 & \text { otherwise. }\end{array}\right.$. 
Also, the trial tangential stress is calculated as

$t_{\mathrm{T} \alpha, n+1}^{\mathrm{trial}, \mathrm{I}}=t_{\mathrm{T} \alpha, n}^{\mathrm{I}}+\epsilon_{\mathrm{T}} a_{\alpha \beta}^{\mathrm{I}}\left(\xi_{n+1}^{\beta, \mathrm{I}}-\xi_{n}^{\beta, \mathrm{I}}\right)$

where $N_{\mathrm{c}}$ is the total number of control points that satisfy $g_{\mathrm{N}}^{\mathrm{I}} \leq 0$ and $a_{\alpha \beta}^{\mathrm{I}}$ is evaluated as

$a_{\alpha \beta}^{\mathrm{I}}=\frac{\left\langle a_{\alpha \beta} R^{\mathrm{I}}\right\rangle}{\left\langle R^{\mathrm{I}}\right\rangle}$.

Furthermore, by checking the slip condition with this trial tangential stress, we compute the actual tangential stress with the formula

$t_{\mathrm{T}, \mathrm{n}+1}^{\mathrm{I}}= \begin{cases}t_{\mathrm{T}, n+1}^{\text {trial,I }} & \text { if } f_{\mathrm{s}, n+1}^{\text {trial }, \mathrm{I}} \leq 0 \\ -\mu_{\text {fric }}\left|p_{\mathrm{N}, n+1}^{\mathrm{I}}\right| \frac{t_{\mathrm{T}, n+1}^{\text {trial }, \mathrm{I}}}{\left\|\boldsymbol{t}_{\mathrm{T}, n+1}^{\mathrm{trial}, \mathrm{I}}\right\|} & \text { otherwise. }\end{cases}$

as derived in Eq. (27). Finally, with these averaged quantities at hand, virtual work (22) associated with contact as evaluated as

$C_{\mathrm{c}}=\sum_{\mathrm{I}=1}^{N_{\mathrm{c}}}\left(\delta g_{\mathrm{N}}^{\mathrm{I}} p_{\mathrm{N}}^{\mathrm{I}}+\delta \xi^{\alpha, \mathrm{I}} t_{\mathrm{T}, \alpha}^{\mathrm{I}}\right)\left\langle R^{\mathrm{I}}\right\rangle$.

\section{Constraint conditions for in-plane UC and sub-UC}

NPT is the procedure to solve the set of governing Eqs. (5) and (6) along with appropriate constitutive laws of the constituent materials for the mesoscopic fist Piola-Kirchhoff stress $\boldsymbol{P}$, deformation gradient $\boldsymbol{F}$ and displacement $\boldsymbol{w}$, under the constraint conditions (3) and (4). Then, the test results are utilized to compute Macro-PK1-RS by Eqs. (7) and (8). Although the formulation seems to be completed, the solution of the mesoscopic equilibrium condition (6) is indeterminate due to the periodicity constraints, which suppresses three rigid body rotations, but allows three rigid body translations. To suppress them, a single point in the in-plane UC is fully constrained not to move in this study. However, it is necessary to be careful for the choice of this single point, as the additional constraint should not interfere with the contact conditions and constraints Eqs. (3) and (4).

In general, the minimum periodic unit is chosen for a representative volume element (RVE) in computational homogenization. In fact, we identify the in-plane unit cell of a typical dry woven fabrics as shown in Fig. $4 \mathrm{~b}$ as in the previous studies $[40,41,51]$. However, this in-plane unit cell can be divided into four regions as shown in Fig. 4b, which has the following characteristics:

All the one quarter regions, $\mathrm{A}, \mathrm{B}, \mathrm{C}$ and $\mathrm{D}$, have the same geometrical pattern; each one is transformed to another with use of rotational symmetry with respect to the axis parallel to $Y_{1}{ }^{-}, Y_{2}$ - or $Y_{3}$-axis. For example, Region A yields Region B by $180^{\circ}$ rotational symmetry transformation with respect to the axis parallel to $Y_{2}$ axis, while A and D becomes identical when we apply $180^{\circ}$ rotational symmetry transformation with respect to the axis parallel to $Y_{3}$-axis

Therefore, instead of the standard in-plane unit cell, the inplane sub-unit cell (sub-UC) as shown in Fig. 4c is selected as the minimum unit in this study. This kind of selection of RVEs is not only advantageous in terms of computational costs as motivated in Reference [52], but also makes NPTs involving frictional contact problems of multiple bodies stable under
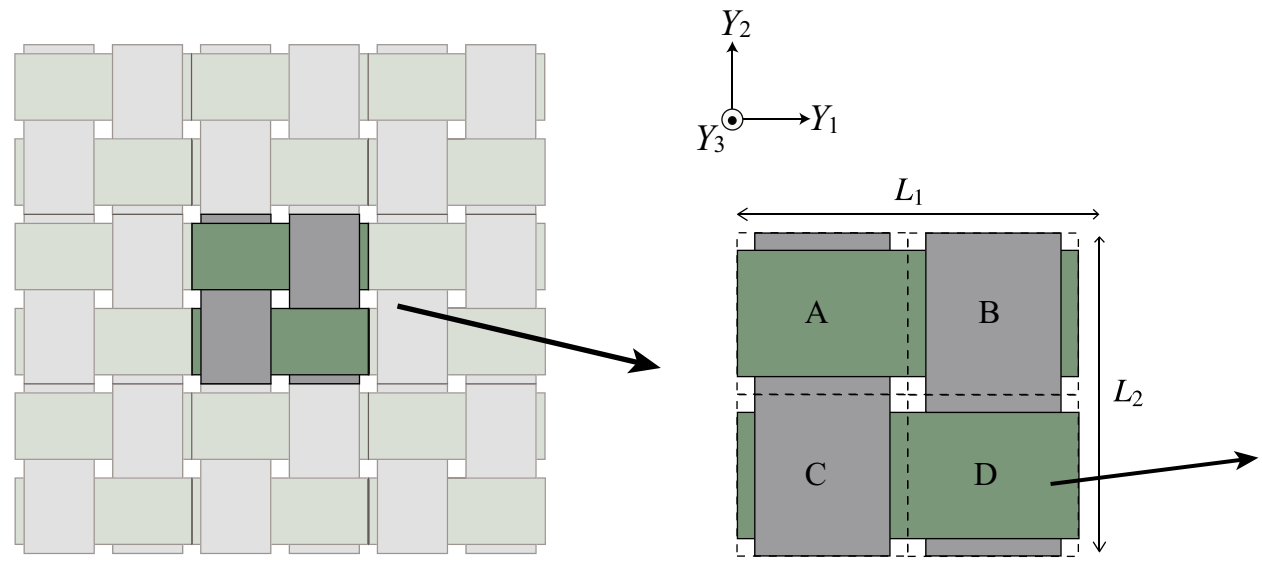

(a) Overall structure of dry woven-fabric

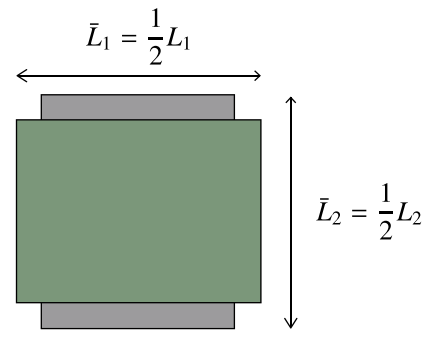

(c) In-plane sub-unit cell

Fig. 4 Definition of in-plane unit cell and sub in-plane unit cell 


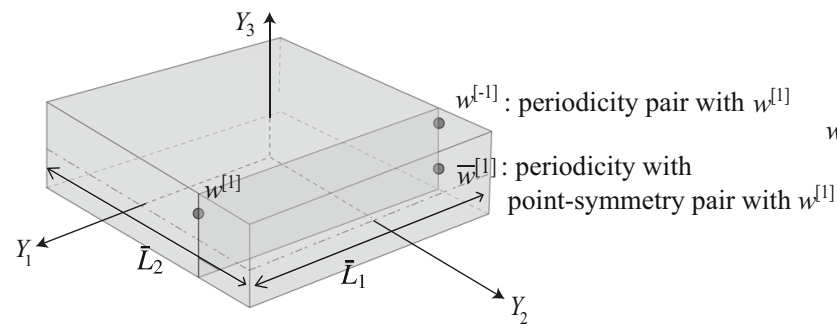

(a) Periodicity and anti-periodicity pairs

Fig. 5 Constraint conditions for in-plane sub-unit cell

periodicity constraints. However, the in-plane sub-UC must be provided with appropriate constraint conditions on its boundaries to kinematically equivalent to the original inplane UC.

At first, as a matter of notational convenience, let us rewrite Eqs. (3) and (4) as

$\mathcal{G}\left(\boldsymbol{w}^{[J]}, \boldsymbol{w}^{[-J]}\right)=\mathcal{H}^{[J]}\left(\tilde{H}_{n}, L_{J}\right)$,

where $\mathcal{G}$ is defined as

$\mathcal{G}\left(\boldsymbol{w}^{[J]}, \boldsymbol{w}^{[-J]}\right):=\left\{\begin{array}{c}w_{1}^{[J]}-w_{1}^{[-J]} \\ w_{2}^{[J]}-w_{2}^{[-J]} \\ w_{3}^{[J]}-w_{3}^{[-J]}\end{array}\right\}$.

Also, $\mathcal{H}^{[J]}\left(\tilde{H}_{n}, L_{J}\right)(n=1 \sim 6)$ are the $3 \mathrm{D}$ vectors whose components correspond to the expressions on the right-hand sides of Eqs. (3) and (4). To be more specific, for constraint conditions (3) and (4), $\mathcal{H}^{[J]}$ is defined as

$\mathcal{H}^{[1]}=\left\{\begin{array}{c}\left(\tilde{H}_{1}+Z_{3} \tilde{H}_{4}\right) L_{1} \\ \frac{1}{2}\left(\tilde{H}_{3}+Z_{3} \tilde{H}_{6}\right) L_{1} \\ -\frac{1}{2} Z_{2} \tilde{H}_{6} L_{1}\end{array}\right\}$,
$\mathcal{H}^{[2]}=\left\{\begin{array}{c}\frac{1}{2}\left(\tilde{H}_{3}+Z_{3} \tilde{H}_{6}\right) L_{2} \\ \left(\tilde{H}_{2}+Z_{3} \tilde{H}_{5}\right) L_{2} \\ -\frac{1}{2} Z_{1} \tilde{H}_{6} L_{2}\end{array}\right\}$.

In consideration of the above-mentioned geometrical characteristics of the in-plane sub-UC, the following constraint condition can be established:

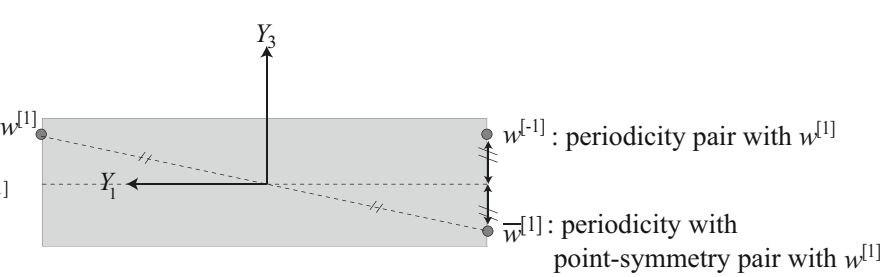

(b) Cross-section of in-plane sub-unit cell

$\overline{\mathcal{G}}\left(\boldsymbol{w}^{[J]}, \boldsymbol{w}^{[-J]}\right)=\mathcal{H}^{[J]}\left(\tilde{H}_{n}, \bar{L}_{J}\right)$,

where $\overline{\mathcal{G}}$ has been defined as

$\overline{\mathcal{G}}\left(\boldsymbol{w}^{[J]}, \overline{\boldsymbol{w}}^{[-J]}\right):=\left\{\begin{array}{c}w_{1}^{[J]}-\bar{w}_{1}^{[J]} \\ w_{2}^{[J]}-\bar{w}_{2}^{[J]} \\ w_{3}^{[J]}+\bar{w}_{3}^{[J]}\end{array}\right\}$.

Here, $\overline{\boldsymbol{w}}^{[J]}=\left\{\bar{w}_{1}^{[J]}, \bar{w}_{2}^{[J]}, \bar{w}_{3}^{[J]}\right\}^{\mathrm{T}}$ is the displacement vector on the point that can be identified by point-symmetric transformation of a point on the $Y_{J} Y_{3}$ plane containing these points; see Fig. 5. Also, $\bar{L}_{\alpha}$ in the right-hand side of Eq. (47) is the width of an in-plane sub-UC as shown in Fig. 5; that is, $\bar{L}_{\alpha}$ is half size of $L_{\alpha}$ as depicted in Fig. 4. Note here that only the $Y_{3}$-component is set to be anti-periodic. These settings of an in-plane sub-UCs would suppress in part numerical instabilities due to rigid body motions of fiber bundles contacting with each other. In fact, each bundle can move independently in the absence of frictional contacts between fiber bundles. Thus, as the number of rigid motion modes of an in-plane UC having four fiber bundles is twice as that of an in-plane sub-UC having two fiber bundles, the former is less stable than the latter.

On the other hand, let us consider the situation as shown in Fig. 6a that illustrates the in-plane shear deformation of the in-plane sub-UCs. Here, the frictional contacts between upper and lower fiber bundles in the lighter and darker regions cannot be properly analyzed, since possible contact surfaces are outside the analysis domain of the in-plane sub-UC centered in this figure. Therefore, we virtually place eight sub-UCs around the in-plane sub-UC as shown in Fig. 6b, and transfer all the data in the in-plane sub-UC to these 'virtual' sub-UCs. The displacement vectors of the virtual sub-UCs (indicated by subscript VUCx with $\times$ being the virtual domain number) are related to those of the centered in-plane sub-UC (indicated by subscript 'UC') as 


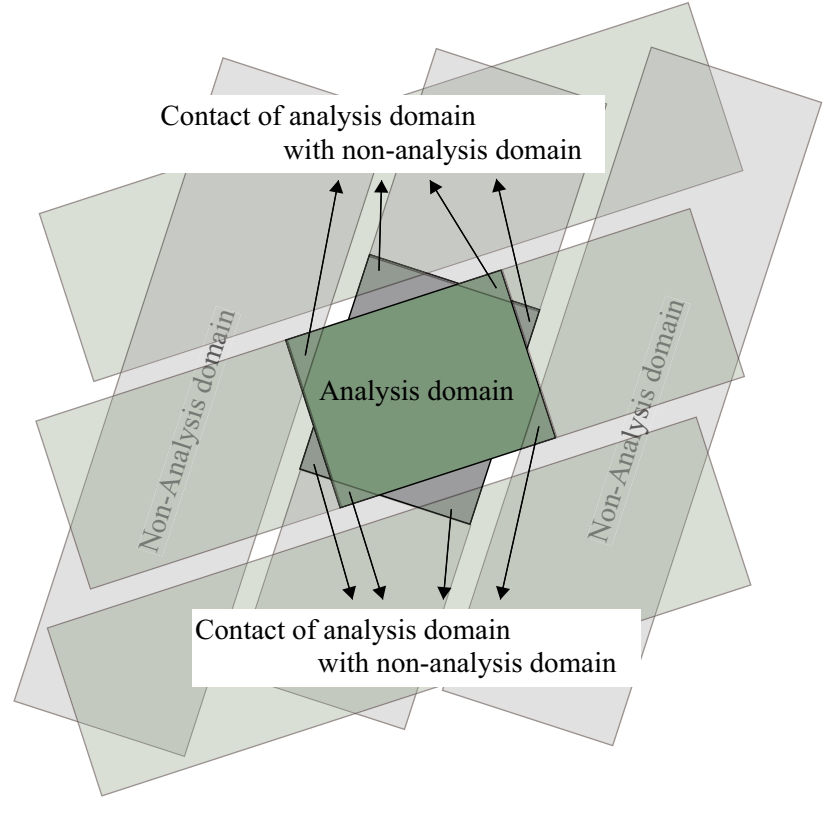

(a) Analysis domain srrounded by non-analysis domains

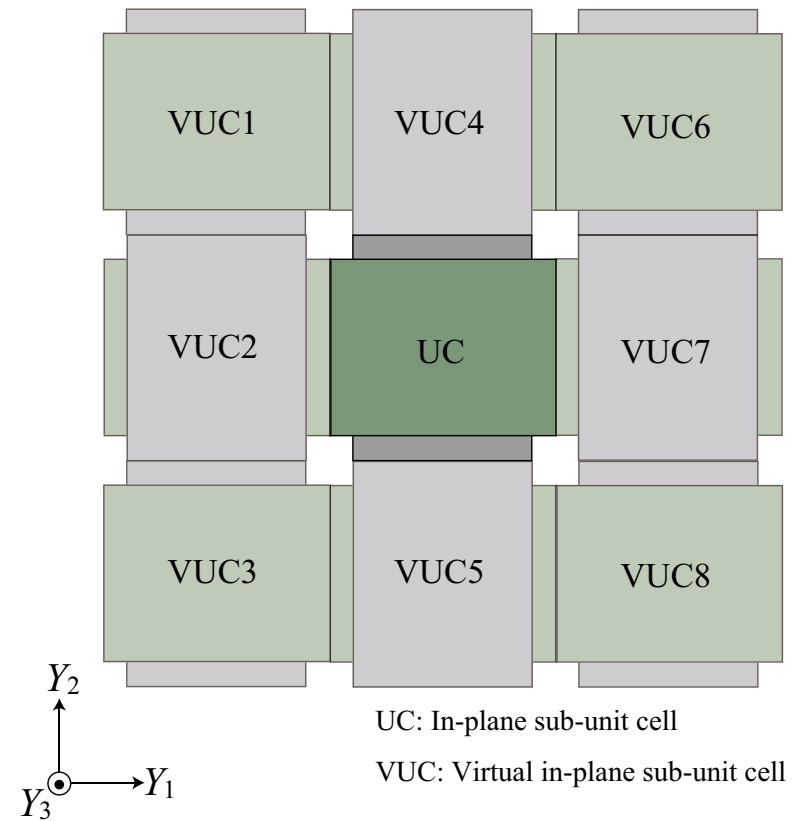

(b) Virtual in-plane UCs placed arround in-plane UC

Fig. 6 Treatment of in-plane unit cell subject to in-plane shear deformation

Fig. 7 Initial IGA model of in-plane sub-unit cell for weaving simulation of dry woven fabrics at meso-scale

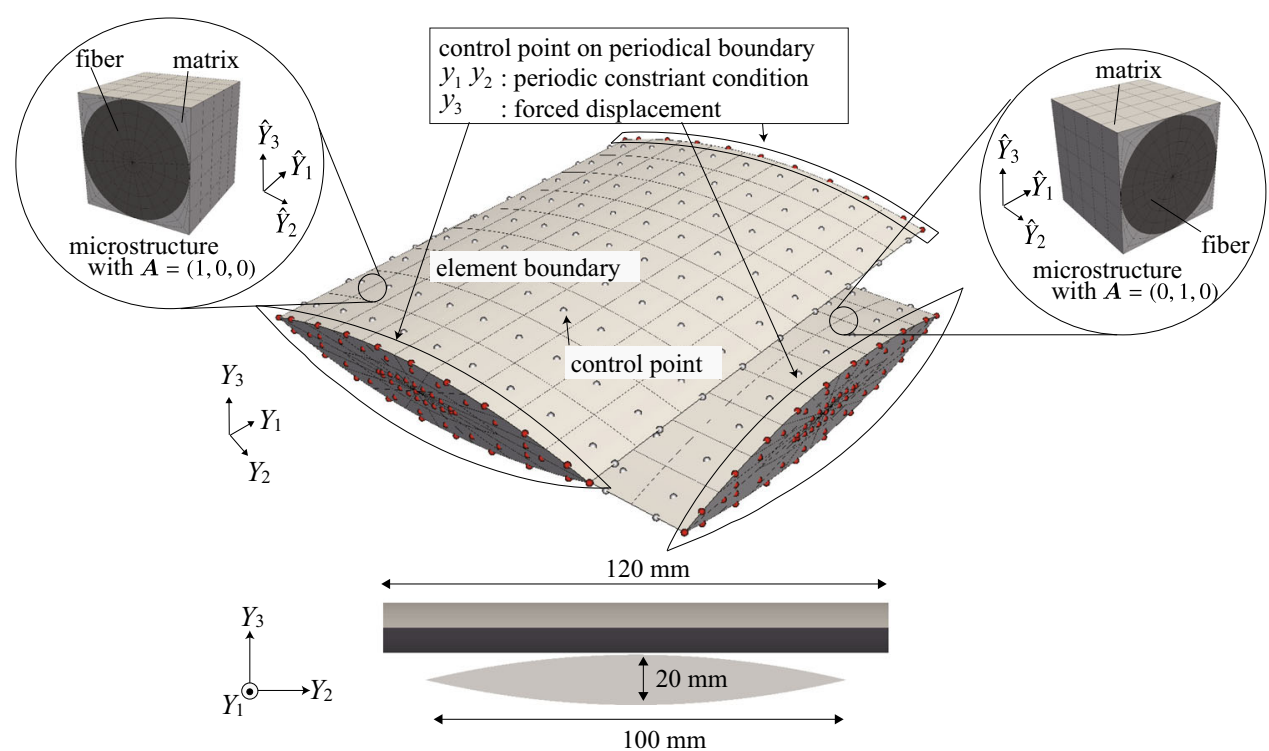

Table 1 Identified material parameters of fiber bundle (MPa)

\begin{tabular}{llllll}
\hline$\mu_{\text {iso }}$ & $\lambda_{\text {iso }}$ & $a_{\text {trn }}$ & $b_{\text {trn }}$ & $c_{\text {trn }}$ & $d_{\text {trn }}$ \\
\hline 3.2675 & 3.9042 & -2.3451 & 16.167 & 4.7881 & -6.4613
\end{tabular}

\section{Generating in-plane sub-unit cell}

We generate an initial state of the in-plane sub-UC of a dry woven fabric, which will be used for NPTs in the next section. After the mesoscopic material model used for the 

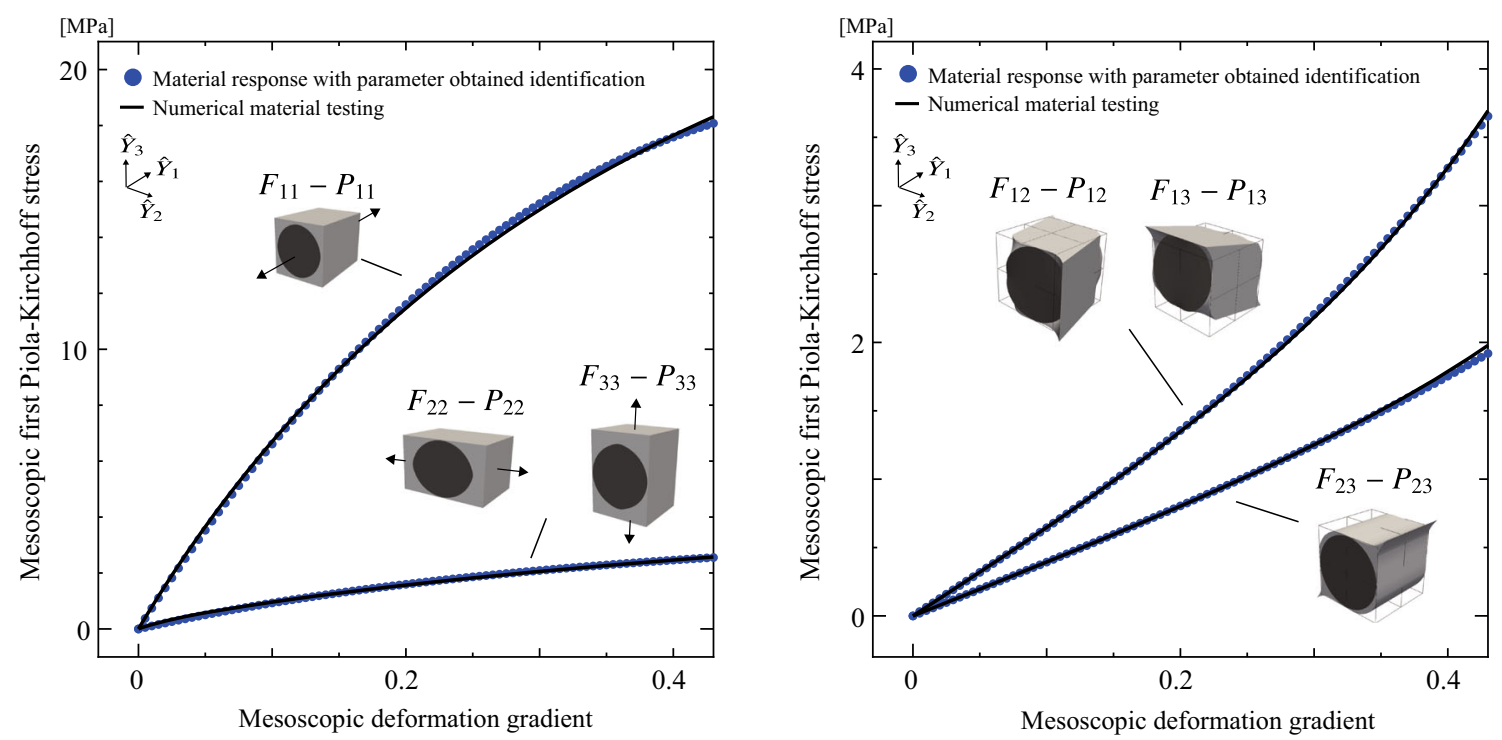

Fig. 8 Results of NMTs with mesoscopic deformations being imposed: tensile loading in the $\hat{Y}_{1}, \hat{Y}_{2}$ and $\hat{Y}_{3}$ directions (left), and shear loading in the $\hat{Y}_{1} \hat{Y}_{2}$ and $\hat{Y}_{2} Z_{3}$ and $\hat{Y}_{3} \hat{Y}_{1}$ planes (right)

Fig. 9 Numerical results of weaving simulation in-plane sub-unit cell

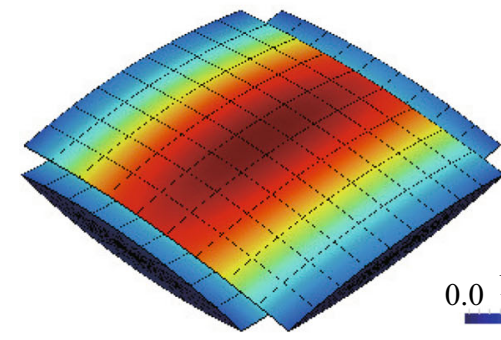

0 Norm of displacement [mm] 5.0

(a) Deformed configuration with displacement distribution

in-plane sub-unit cell

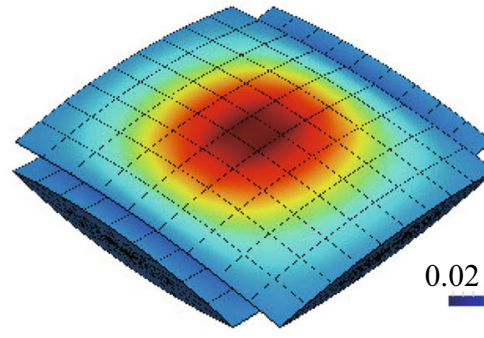

vons-Mises stress [MPa]

(b) Deformed configuration with von-Mises stress distribution

fiber bundles in the cell is presented, its material parameters are determined by the numerical material testing (NMT) within the framework of nonlinear homogenization. Then, the weaving process is performed on non-deformed straight fiber bundles so that the natural initial geometry of the inplane sub-UC along with the initial stress caused by bending of fiber bundles would be obtained.

\subsection{Setting of meso-scale analysis}

Figure 7 shows the straight fiber bundles before a weaving process. Each fiber bundle is composed of a number of fibers impregnated in resin matrix, which are assumed be arranged periodically in the transverse directions. Representative periodic microstructures, i.e., unit cells, are also depicted in the same figure, and possess their own fiber orientations. 


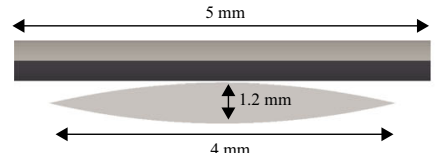

cross section of employed IGA model in case (a)

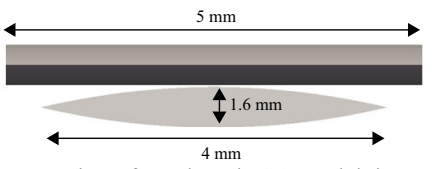

cross section of employed IGA model in case (b)

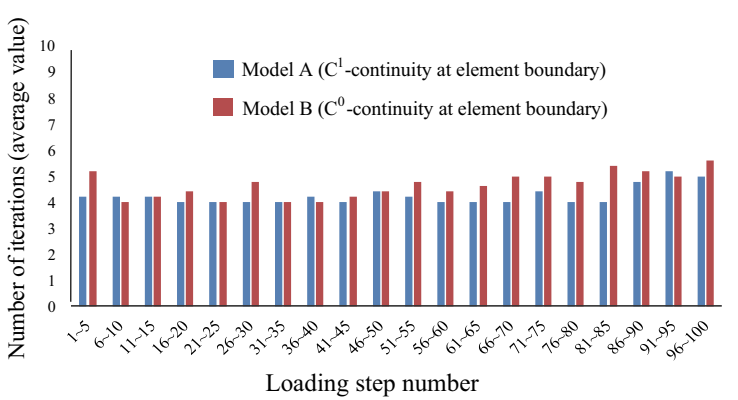

(a) KTS (thickness: $1.2 \mathrm{~mm}$ )

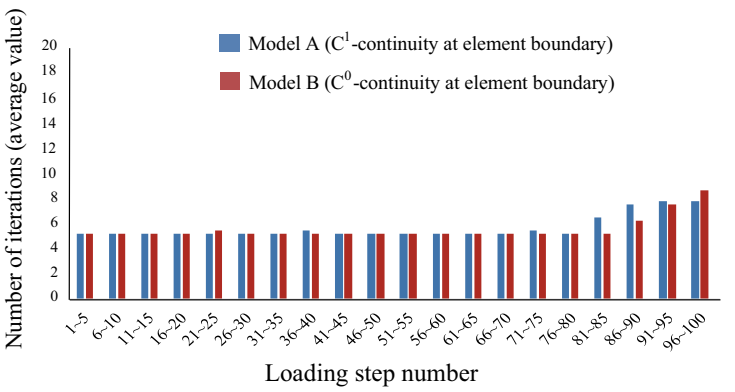

(c) Mortar-based KTS (thickness: $1.2 \mathrm{~mm}$ )

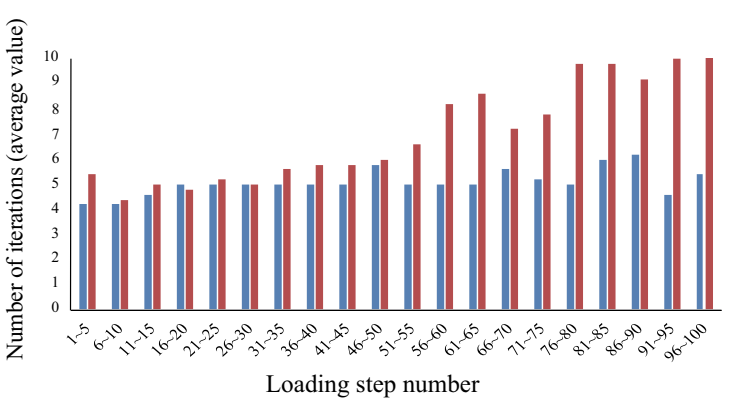

(b) KTS (thickness: $1.6 \mathrm{~mm}$ )

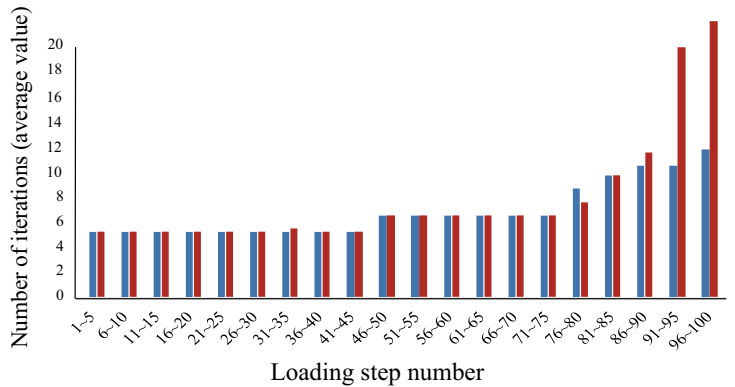

(d) Mortar-based KTS (thickness: $1.6 \mathrm{~mm}$ )

Fig. 10 Mean number of Newton-Raphson iterations in every consecutive 5 loading steps

Fig. 11 Results of NPT with macroscopically tensile loading in the $Y_{1}$ direction

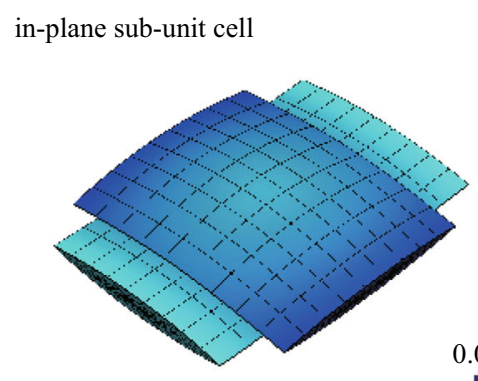

Norm of displacement [mm]

overall structure

(a) Deformed configuration with displacement distribution

in-plane sub-unit cell

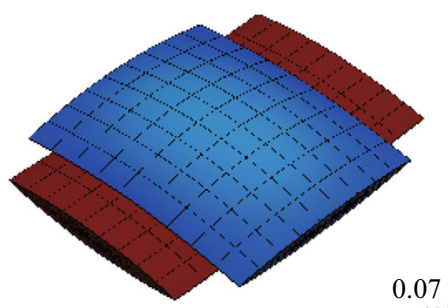

vons-Mises stress [MPa]

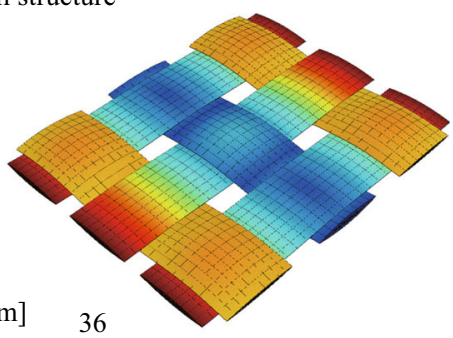

overall structure

(b) Deformed configuration with stress distribution 


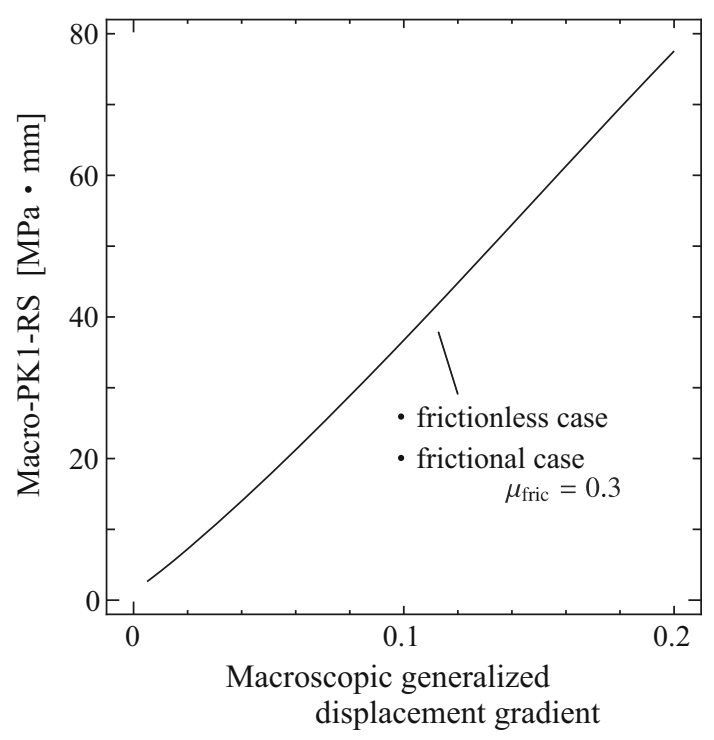

Fig. 12 Relationships between Macro-PK1-RS's and macroscopic generalized displacement gradients in response to macroscopically tensile loading in the $Y_{1}$ direction

In general, the mesoscopic material response of the fiber bundle is assumed to be transversely isotropic. In this study, we employ the following strain energy function proposed by Bonet [53] to represent the transversely isotropic hyperelastic behavior:

$$
\Psi=\Psi_{\text {iso }}+\Psi_{\text {trn }}
$$

$$
\begin{aligned}
\Psi_{\text {iso }}= & \frac{\mu_{\text {iso }}}{2}\left(I_{1}-3\right)-\mu_{\text {iso }} \ln J+\frac{\lambda_{\text {iso }}}{2}(\ln J)^{2}, \\
\Psi_{\text {trn }}= & \left\{a_{\mathrm{trn}}+b_{\mathrm{trn}} \ln J+c_{\mathrm{trn}}\left(I_{4}-1\right)\right\}\left(I_{4}-1\right) \\
& -\left(\frac{1}{2} a_{\mathrm{trn}}+d_{\mathrm{trn}} \ln J\right)\left(I_{5}-1\right) .
\end{aligned}
$$

Here, $\Psi_{\text {iso }}$ and $\Psi_{\text {trn }}$ are the strain energy densities of isotropic components and transversely components, respectively. Also, $I_{1}, I_{4}$ and $I_{5}$ are the invariants respectively defined as

$$
I_{1}=\operatorname{tr} \boldsymbol{C}, \quad I_{4}=\boldsymbol{A} \cdot \boldsymbol{C A}, \quad I_{5}=\boldsymbol{A} \cdot \boldsymbol{C}^{2} \boldsymbol{A},
$$

where $\boldsymbol{A}$ is the unit vector directed to the longitudinal direction of fibers in the initial configuration. It is noted here that the original model in [53] does not have the term $d_{\mathrm{trn}} \ln J\left(I_{5}-1\right)$ in $(52)$, which is added to improve the identification accuracy of the material parameters determined from NMT results.

Following the formal procedure of the nonlinear homogenization [11], we perform NMTs on the unit cell shown in Fig. 7 to identify the material parameters $\mu_{\text {iso }}, \mu_{\text {iso }}, a_{\text {trn }}, b_{\text {trn }}$, $c_{\text {trn }}$ and $d_{\text {trn }}$ in Eq. (52). The standard neo-Hookean hyperelastic constitutive model is employed for both the fiber and matrix in the unit cell, in which Young's moduli of fiber and matrix are set at $E_{\mathrm{f}}=100 \mathrm{MPa}$ and $E_{\mathrm{m}}=1 \mathrm{MPa}$, respectively, and Poisson ratio is set at 0.3 for both of the constituents. The fiber and matrix are assumed to be per-
Fig. 13 Results of NPT with macroscopically shear loading in the $Y_{1} Y_{2}$ plane in-plane sub-unit cell

overall structure

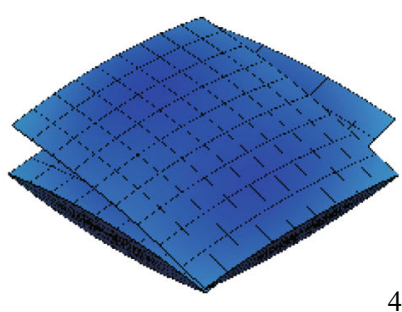

4.5 Norm of displacement [mm]

25.1

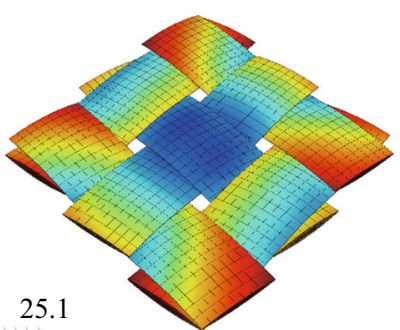

(a) Deformed configuration with displacement distribution

in-plane sub-unit cell

overall structure

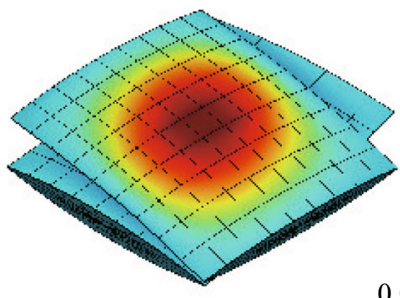

0.05 vons-Mises stress $[\mathrm{MPa}]$

1.6

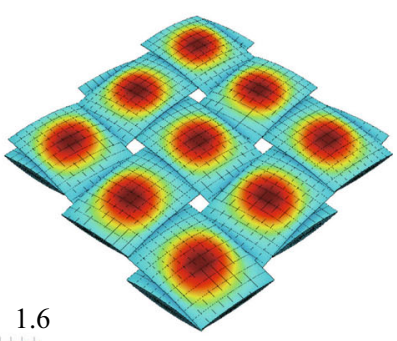

(b) Deformed configuration with stress distribution 


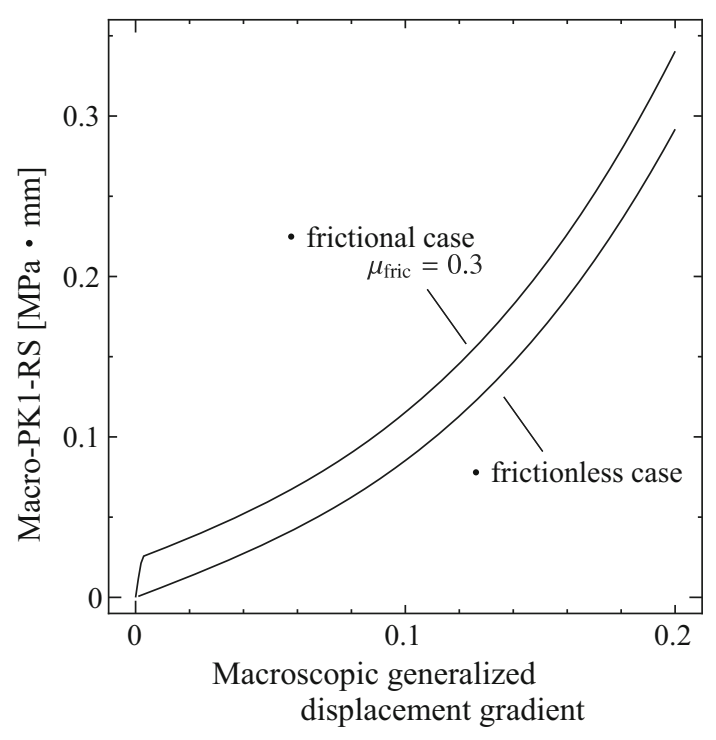

Fig. 14 Relationships between Macro-PK1-RS's and macroscopic generalized displacement gradients in response to macroscopically shear loading in the $Y_{1} Y_{2}$ plane

fectly bonded and the volume fraction of the fiber is set at $77 \%$. Three separate tensile and three shear modes of macroscopic deformations are imposed as constraint conditions for the periodic pairs of boundary nodes in the NMTs. Then, the material parameters in the assumed constitutive model, which have been determined by an optimization scheme to be in accordance with the NMT results, are presented in Table 1. Figure 8 provides the comparison between the identified curves with these parameters and those obtained by the NMTs. As can be seen from the plots, in which the mechanical behavior of fiber bundle exhibits typical anisotropy according to the deformation modes, the identification was successful.

For the meso-scale frictional contact between fiber bundles, the friction coefficient is set to $\mu_{\text {fric }}=0.3$, and the penalty parameters for the normal and tangential contacts, $\epsilon_{\mathrm{N}}$ and $\epsilon_{\mathrm{T}}$, are set to be 100 .

\subsection{Initial state of in-plane sub-unit cell}

A weaving process of the undeformed two fiber bundles in Fig. 7 is simulated to obtain the initial state of the IGA model of the in-plane sub-UC. The $Y_{3}$-components of the displacements of the red-colored control points on a boundary surface are enforced to coincide with those on the opposite boundary surface so that both of the fiber bundles are bent to form a woven shape. At the same time, the pairs of the $Y_{1^{-}}$and $Y_{2}$ - components on the boundary surfaces are constrained as $w_{1}^{[J]}=w_{1}^{[-J]}$ and $w_{2}^{[J]}=w_{2}^{[-J]}$. During this weaving simulations, the fiber bundles contact with each other and are stressed by bending.

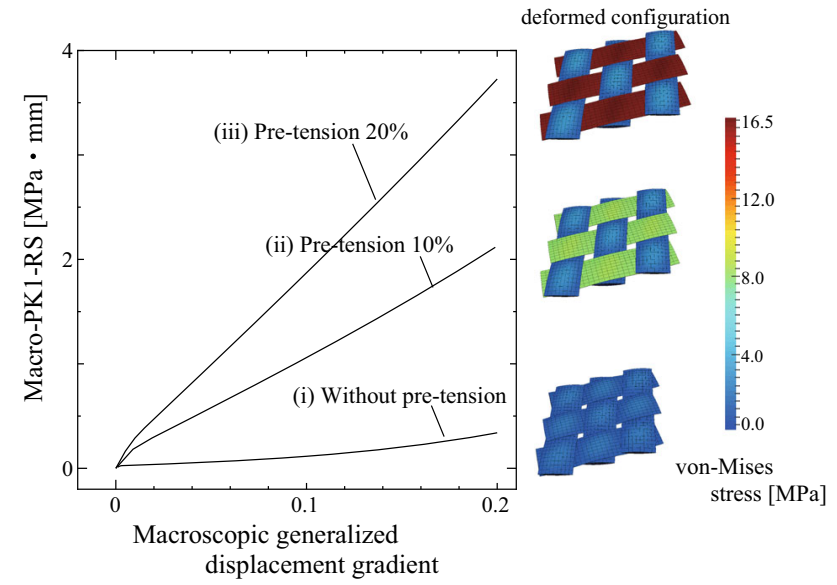

Fig. 15 Relationships between Macro-PK1-RS's and macroscopic generalized displacement gradients in response to macroscopically shear loading in the $Y_{1} Y_{2}$ plane after three different cases of pre-tensile loading; (i) case without pre-tension, (ii) case with $10 \%$ pre-tension and (iii) case with $20 \%$ pre-tension to examine the influence of pre-tension

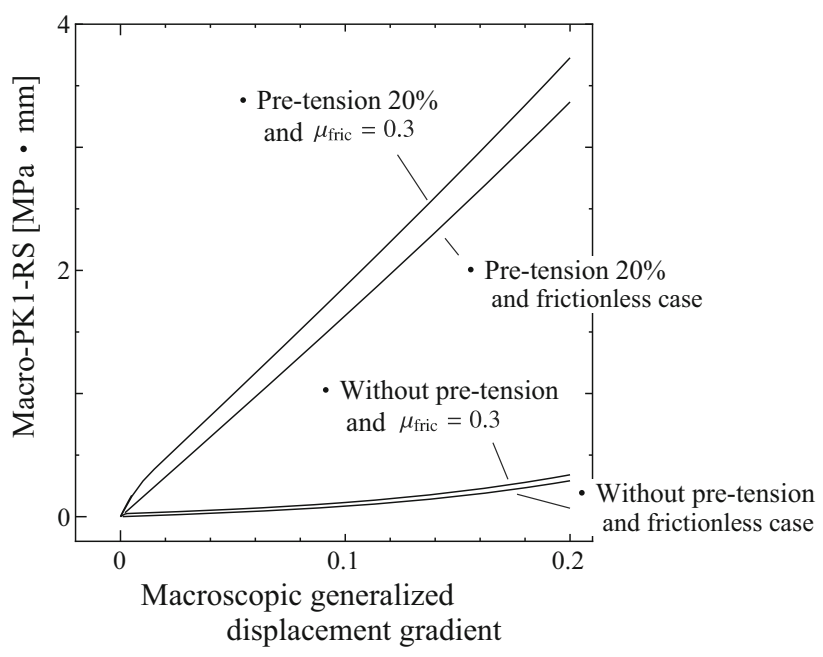

Fig. 16 Influence of friction coefficient on relationships between Macro-PK1-RS's and macroscopic generalized displacement gradients in response to macroscopic shear loading in the $Y_{1} Y_{2}$ plane applied to the in-plane sub-UCs subjected to $20 \%$ pre-tension and without pre-tension

After obtaining the woven forms of the fiber bundles sustaining the initial stress due to bending, we solve the self-equilibrium problem of the in-plane sub-UC with the constraint conditions (44) with $\mathcal{H}^{[J]}=\mathbf{0}$ to release the over-constraining imposed during weaving process. Figure 9 shows the deformed configurations of the in-plane unit cell and of the overall structure containing virtual sub-UCs along with the norm of displacement and the von-Mises stress occurred in the weaving process. 
Fig. 17 Convergence trend in relationships between Macro-PK1-RS's and macroscopic generalized displacement gradients in response to macroscopically shear loading in the $Y_{1} Y_{2}$ plane with different penalty parameters

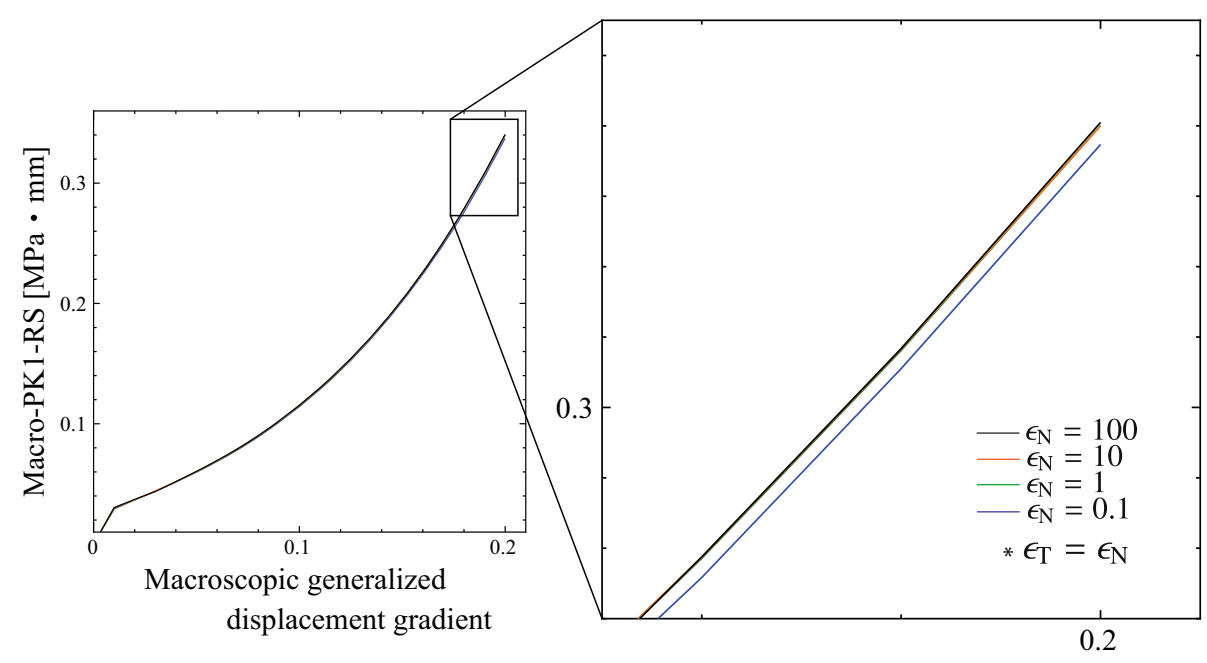

Fig. 18 Convergence trend in relationships between Macro-PK1-RS's and macroscopic generalized displacement gradients in response to macroscopically shear loading in the $Y_{1} Y_{2}$ plane with different IGA mesh sizes

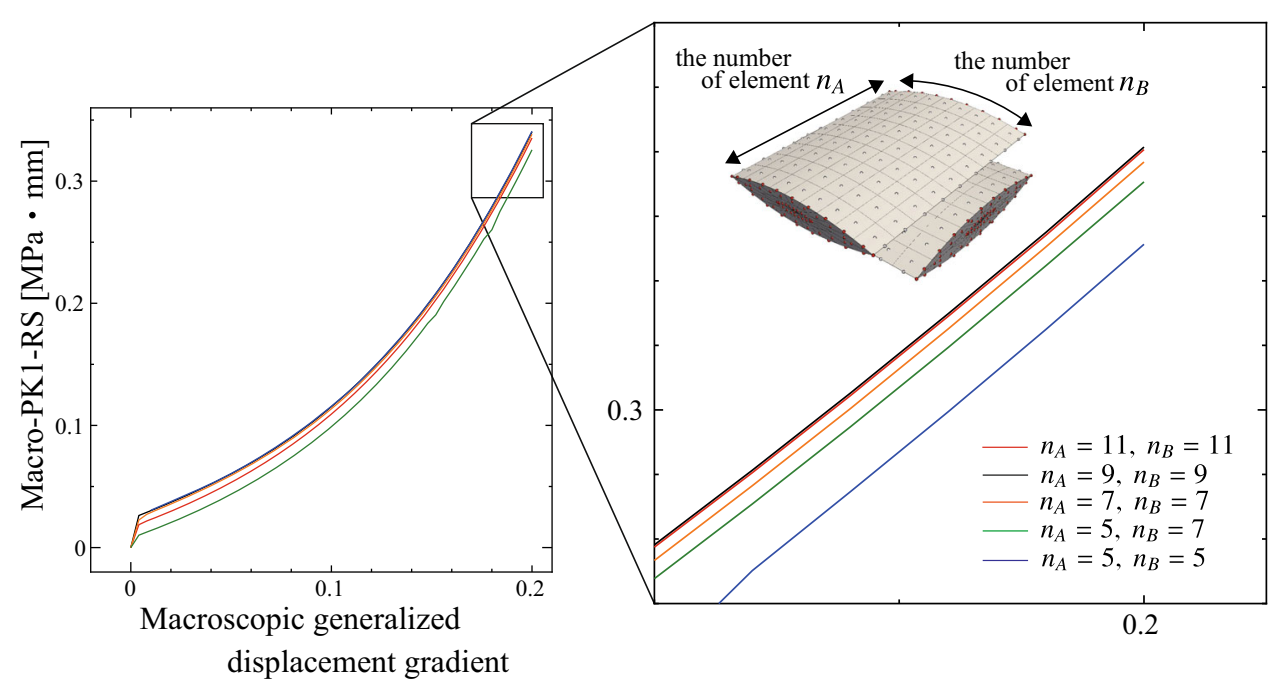

\section{Representative numerical examples}

In order to discuss the validity and capability of the present approach, we carry out several numerical analyses of the inplane sub-UC generated in the previous section. Prior to the characterization of the macroscopic mechanical behavior of dry woven fabrics by NPTs involving frictional contact, a numerical test for computational efficiency is conducted to demonstrate the performance of the IGA with the NURBS basis functions. Then, the macroscopic in-plane and out-ofplane mechanical behaviors are characterized by the IGAbased NPT. In particular, we are concerned with the effect of pre-tension on the macroscopic mechanical behavior, which is supposed to be realized by 'inelastic' material response.

\subsection{Numerical test for computational efficiency}

In IGA with high-order NURBS basis functions, high convergence performance is expected in solving frictional contact problems because of the smooth geometrical representation of contact surfaces, which is also reflected in the continuity of discretized displacement. In this section, we verify such a convergence characteristic in terms of the number of Newton-Raphson (NR) iterations for the 'global' equilibrium problem with the following two IG models that have the same geometry and the same number of elements:

- Model A having $C^{1}$ continuity on element boundaries,

- Model B having $C^{0}$ continuity on element boundaries, which is equivalent to the standard FE model.

For each of the models, we have prepared two different geometries of the fiber bundles to investigate the effect of size of contact areas; one has a thickness of $1.2 \mathrm{~mm}$ and the other of $1.6 \mathrm{~mm}$ as shown in the upper portion of Fig. 10, while all the other sizes are identical. An in-plane shear deformation of $50 \%$ macroscopic displacement gradient is enforced on the in-plane sub-UC with 100 loading steps. It should be noted 
that the active set on the contact area is continuously updated during the NR iterative process. To examine the stability associated with the determination of contact areas, friction is not considered in NPTs in this subsection. The degrees of convergence performance are compared in terms of the number of iterations until the relative errors in 'global' equilibrium states become smaller than the prescribed tolerance value. In this study, it is set to be $10^{-7}$.

Figure 10 shows the mean number of NR iterations in every consecutive 5 loading steps with the standard KTS and mortar-based KTS method. In all the cases tested here, it was consistently observed that $C^{1}$ continuity delivers a smaller total number of iterations to simulation completion than $C^{0}$ continuity. This indicates an advantage of employing B-splines and NURBS at the mesoscopic level in the context of the standard KTS method (Fig. 10a, b). However, in view of additional numerical robustness criteria mentioned earlier, it is important to take one step further and embed this method within a mortar-based setting. The transition from the standard to the mortar-based setting will by itself also influence the total number of iterations and this influence on the overall performance may vary for different mortar approaches. Nevertheless, for the particular mortar approach pursued in this work, it was again observed that $C^{1}$ continuity retains the advantage over $C^{0}$ continuity in total number of iterations to simulation completion as shown in Fig. 10c, d.

In the following sections, apart from Models A and B, we will exclusively use the model prepared in Sect. 6.2, whose initial configuration is provided in Fig. 7.

\subsection{Macroscopic in-plane characteristics}

In this section, we carry out NPTs for the two in-plane deformation modes realized by Eqs. (3) and (4). One is the in-plane elongation in the $Y_{1}$ direction and the other is the in-plane shear mode.

First, a macroscopic in-plane elongation of $20 \%$ displacement gradient is imposed on in-plane sub-UC in the $Y_{1}$ direction. Figure 11 shows the deformed configurations with distributions of norm of displacement and von-Mises stress. The unidirectional state for each fiber bundle can be recognized from the figures. Figure 12 shows the relationships between the macroscopic displacement gradient $\tilde{H}_{1}$ and the corresponding component of Macro-PK1-RS for frictionless and frictional cases. As can be expected, the effect of frictional contact seems to be indistinctive in the macroscopic deformation characteristics, as the curves are almost linear.

Next, we conduct NPTs for a macroscopical in-plane shear deformation of $20 \%$ displacement gradient with $\tilde{H}_{1}$ and $\tilde{H}_{2}$ being zeros. Figure 13 shows the deformed configurations with the distributions of the norm of displacement and von-Mises stress. High von-Mises stress occurring on fiber bundles is mostly due to the bending of fiber bundles, as can be recognized in comparison with that of Fig. 10, which provides the initial state of the in-plane sub-UC. In fact, the mesoscopic shear stress is not increased significantly, since the fiber bundles themselves are not subjected to shear deformation. In other words, the macroscopic shear deformation has been realized by the large rotation of fiber bundles about $Y_{3}$-axis. Figure 14 shows the relationships between the macroscopic displacement gradient $\tilde{H}_{3}$ and the corresponding component of Macro-PK1-RS for frictionless and frictional cases. It can be seen from the figure for the frictional case that Macro-PK1-RS increases linearly up to $1 \%$ of the macroscopic displacement gradient, but becomes gentle subsequently. This is due to the fact that almost all the Gaussian quadrature points in the active set of contact meet the Coulomb slip criteria and slip with friction. In fact, this macroscopic 'inelastic' material behavior is not present in the frictionless case and can be substituted by an elastic-plastic material.

\subsection{Influence of pre-tension on macroscopic in-plane shear characteristics}

In view of actual draping processes, the effects of pre-tension is investigated in this subsection. For that purpose, the macroscopic displacement gradient $\tilde{H}_{1}$ is first imposed on the in-plane sub-UC so that the fiber bundle in parallel to the $Y_{1}$ axis undergoes initial stress as a result of elongation. Three different amounts of pre-tension by $\tilde{H}_{1}$ are imposed; initial elongations of $10 \%$ and $20 \%$, and without pre-tensioning, which is the same situation as the frictional case in the previous subsection. Then, the macroscopic in-plane shear deformation of $20 \%$ displacement gradient $\tilde{H}_{3}$ is imposed for each in-plane sub-UC subjected to the pre-tension-induced initial stress, while all the other components remain fixed. Friction is active for all the analysis cases.

Figure 15 shows the obtained relationships between the macroscopic displacement gradient $\tilde{H}_{3}$ and the corresponding component of Macro-PK1-RS vector. All the tendencies are similar to the frictional case in Fig. 14, but the larger the amount of pre-tension, the larger the inclination. This is a natural response as the pre-tension generally causes the fiber bundles to stiffen, as can also be observed in actual experiments; see Reference [48]. But, more importantly, the transition from the behavior caused by sticking on the contact surface in the beginning of shear deformation to the following behavior with slipping becomes smooth as the amount of pre-tension increases. This must be due to the fact that the number of Gaussian quadrature points in the active set on the contact area and the variation in normal pressure at these points become large, as the amount of pre-tension increases.

Finally, to see the effect of friction on the degree of the above-examined influence of pre-tension, we carry out an additional numerical analysis with the same amount of 
pre-tension as in case (iii), but without friction. Figure 16 illustrates the comparison of the result with that of case (iii) shown in Fig. 15 and those presented in Fig. 14. As can be seen from the figure, the larger the friction effect, the more significant the influence of pre-tension. In fact, the increase in Macro-PK1-RS by the presence of the friction effect in the case with $20 \%$ pre-tension is much larger than that without pre-tension.

\subsection{Verification analyses}

In order to verify the appropriateness of the magnitudes of penalty parameters $\epsilon_{\mathrm{N}}$ and $\epsilon_{\mathrm{T}}$ and the mesh sizes in the IGA, we conduct convergence studies with different values of penalty parameters and with different sizes of elements.

First, Fig. 17 shows the convergence trend of the MacroPK1-RS's in response to macroscopically shear deformations in the $Y_{1} Y_{2}$ plane in the NPT with $0.1,1,10$ and 100 . Here, the value 100 was the same as that employed in the NPT in the previous subsections. As can been seen from this figure, the the curves of the macro-PK1-RS's converge with increasing penalty factors. In particular, the responses obtained with values 1.0, 10 and 100 are undistinguishable.

Next, Fig. 18 shows the convergence trend of the MacroPK1-RS's with respect to the mesh sizes of IGA. We have tested five IGA meshes with different number of elements by varying $n_{A}$ and $n_{B}$ as indicated in the right figure of Fig. 18. The tested cases are $\left(n_{A}, n_{B}\right)=(11,11),(9,9),(7,7)$, $(5,7)$ and $(5,5)$. Here, the mesh with $\left(n_{A}, n_{B}\right)=(9,9)$ was employed for the NPT in Sects. 7.2 and 7.3. It is confirmed from this figure that the macroscopic response curves converge with the increasing number of elements. To be more specific, the difference between the curves obtained by using the meshes with $\left(n_{A}, n_{B}\right)=(11,11)$ and $(9,9)$ are negligibly small.

In conclusion, the penalty parameter and IG mesh employed in the NPTs presented in Sects. 7.2 and 7.3 are valid for the characterization of the macroscopic mechanical behavior of dry woven fabrics that reflect the mesoscopic frictional contact behavior between fiber bundles.

\section{Concluding remarks}

This study presented an application of the IGA method to numerical plate testing of fiber bundles in a dry woven fabric involving frictional contact behavior at meso-scale. Some numerical examples are presented to demonstrate the performance and capability of the proposed method of IGA-based NPT for characterizing the macroscopic structural responses. Although the method of NPT presented in this paper is just an extension of the linear version previously developed by the authors to finite strain problems, the effectiveness of the IGA applied to the mesoscopic frictional contact problem for in-plane unit cells is confirmed through a series of numerical analyses.

As summarized above, our original goal of this paper has been achieved, but further developments have to be made for two-scale analyses by the application of the de-coupling scheme [11]. In particular, the macroscopic nonlinear mechanical behavior obtained by NPTs is just realized by the relationships between macroscopic resultant stresses and generalized strains, implying that both an in-plane crosssection structure and its constituent materials equivalent to the original composite plate are indeterminate in view of performing decoupled two-scale analyses. In order to carry out macroscopic analyses, we need to determine not only macroscopic constitutive laws that can realize the 'inelastic' material behavior obtained by NPTs, but also a substitution model such as a laminated plate must be assumed as in [54]. These are our next challenges we are addressing.

Acknowledgements This work was supported by CSTI (Cross-ministerial Strategic Innovation Promotion Program) and NEDO (New Energy and Industrial Technology Development Organization) for SIP (Innovative design/manufacturing technologies).

\section{References}

1. Sanchez-Palencia E (1980) Non-homogeneous media and vibration theory. Springer, Berlin

2. Zohdi TI, Wriggers P (2005) An introduction to computational micromechanics, lecture notes in applied and computational mechanics. Springer, Berlin

3. Devries F, Dumontet H, Duvaut G, Léné F (1989) Homogenization and damage for composite structures. Int J Numer Methods Eng 27:285-298

4. Guedes JM, Kikuchi N (1990) Preprocessing and postprocessing for materials based on the homogenization method with adaptive finite element methods. Comput Methods Appl Mech Eng 83:143198

5. Matouš K, Geers MGD, Kouznetsova VG, Gillman A (2017) A review of predictive nonlinear theories for multiscale modeling of heterogeneous materials. Comput Phys 330:192-220

6. Geers MGD, Kouznetsova VG, Matous K, Yvonnet J (2017) Homogenization methods and multiscale modeling: nonlinear problems. In: Stein E, de Borst R, Hughes TJR (eds) Encyclopedia of computational mechanics, 6 volume set, 2nd ed. 2018

7. Terada K, Kikuchi N (1995) Nonlinear homogenization method for practical applications. In: Ghosh S, Ostoja-Starzewski M (eds) Computational methods in micromechanics, AMSE AMD, vol 212, pp $1-16$

8. Terada K, Kikuchi N (2001) A class of general algorithms for multi-scale analyses of heterogeneous media. Comput Methods Appl Mech Eng 190:5427-5464

9. Feyel F, Chaboche J-L (2000) $\mathrm{FE}^{2}$ multiscale approach for modelling the elastoviscoplastic behaviour of long fibre $\mathrm{SiC} / \mathrm{Ti}$ composite materials. Comput Methods Appl Mech Eng 183:309-330

10. Fish J, Yuan Z (2005) Multiscale enrichment based on partition of unity. Int J Numer Methods Eng 62:1341-1359 
11. Terada K, Kato J, Hirayama N, Inugai T, Yamamoto K (2013) A method of two-scale analysis with micro-macro decoupling scheme: application to hyperelastic composite materials. Comput Mech 52:1199-1219

12. Suquet PM (1987) Elements of homogenization theory for inelastic solid mechanics. In: Sanchez-Palencia E, Zaoui A (eds) Homogenization techniques for composite media. Springer, Berlin, pp $193-278$

13. Terada K (2007) Fukugouzairyou no suutizairyousiken no susume sononi. Reinf Plast 53(5):246-253 (in Japanese)

14. Terada K, Inugai T, Hirayama N (2008a) A method of numerical material testing in nonlinear multiscale material analyses. Trans Jpn Soc Mech Eng Ser A 74(744):1084-1094 (in Japanese)

15. Terada K, Inugai T, Hamana H, Miyori A, Hirayama N (2008b) Parameter identification for anisotropic hyperelastic materials by numerical material testing. Trans Jpn Soc Comput Eng Sci 20080024 (in Japanese)

16. Terada K, Hamana H, Hirayama N (2009) A method of viscoelastic two-scale analyses for FRP. Trans Jpn Soc Mech Eng Ser A 75(760):1674-1683 (in Japanese)

17. Cybernet Systems Co. Ltd. (2018) Multiscale.Sim ${ }^{\circledR}$. http://www. cybernet.co.jp/ansys/product/lineup/mul-tiscale/en/multiscale_ $\operatorname{sim} / /$. Accessed 31 May 2018

18. Terada K, Hirayama N, Yamamoto K, Muramatsu M, Matsubara S, Nishi S (2016) Numerical plate testing for linear two scale analyses of composite plates with in-plane periodicity. Int J Numer Methods Eng 105:111-137

19. Goncalves BR, Jelovica J, Romanoff J (2016) A homogenization method for geometric nonlinear analysis of sandwich structures with initial imperfections. Int J Solids Struct 87:194-205

20. Geers MGD, Coenen EWC, Kouznetsova VG (2007) Multi-scale computational homogenization of structured thin sheets. Model Simul Mater Sci Eng 15:393-404

21. Coenen EWC, Kouznetsova VG, Geers MGD (2010) Computational homogenization for heterogeneous thin sheets. Int J Numer Methods Eng 83:1180-1205

22. Cong Y, Nezamabadi S, Zahrouni H, Yvonnet J (2015) Multiscale computational homogenization of heterogeneous shells at small strains with extensions to finite displacements and buckling. Int J Numer Methods Eng 104:235-259

23. Mercatoris BCN, Massart TJ (2011) A coupled two-scale computational scheme for the failure of periodic quasi-brittle thin planar shells and its application to masonry. Int $\mathbf{J}$ Numer Methods Eng 85:1177-1206

24. Hughes TJR, Cottrell JA, Bazilevs Y (2005) Isogeometric analysis: CAD, finite elements, NURBS, exact geometry and mesh refinement. Comput Methods Appl Mech Eng 194:4135-4195

25. Kiendle J, Bletzinger KU, Linhard J, Wüchner R (2009) Isogeometric shell analysis with Kirchhoff-Love elements. Comput Methods Appl Mech Eng 198:3902-3914

26. Benson DJ, Bazilevs Y, Hsu MC, Hughes TJR (2010) Isogeometric shell analysis: the Reissner-Mindlin shell. Comput Methods Appl Mech Eng 199:276-289

27. Buffa A, Sangalli G, Vázquez R (2010) Isogeometric analysis in electromagnetics: B-splines approximation. Comput Methods Appl Mech Eng 199:1143-1152

28. Verhoosel CV, Scott MA, Hughes TJR, Borst R (2011) An isogeometric analysis approach to gradient damage models. Int J Numer Methods Eng 86:115-134

29. Seo YD, Kim HJ, Youn SK (2010) Isogeometric topology optimization using trimmed spline surfaces. Comput Methods Appl Mech Eng 199:3270-3296

30. Dedè L, Borden MJ, Hughes TJR (2012) Isogeometric analysis for topology optimization with a phase field model. Arch Comput Methods Eng 19:427-465
31. Lu J (2011) Isogeometric contact analysis: geometric basis and formulation for frictionless contact. Comput Methods Appl Mech Eng 200:726-741

32. Temizer I, Wriggers P, Hughes TJR (2011) Contact treatment in isogeometric analysis with NURBS. Comput Methods Appl Mech Eng 200:1100-1112

33. Lorenzis LD, Temizer I, Wriggers P, Zavarise GA (2011) Large deformation frictional contact formulation using NURBS-based isogeometric analysis. Int J Numer Methods Eng 87:1278-1300

34. Temizer I, Wriggers P, Hughes TJR (2012) Three-dimensional mortar-based frictional contact treatment in isogeometric analysis with NURBS. Comput Methods Appl Mech Eng 209-212:115-128

35. Dittmann M, Franke M, Temizer I, Hesch C (2014) Isogeometric analysis and thermomechanical mortar contact problems. Comput Methods Appl Mech Eng 274:192-212

36. Corbett CJ, Sauer RA (2015) Three-dimensional isogeometrically enriched finite elements for frictional contact and mixed-mode debonding. Comput Methods Appl Mech Eng 284:781-806

37. Kruse R, Nguyen-Thanh N, Lorenzis LDe, Hughes TJR (2015) Isogeometric collocation for large deformation elasticity and frictional contact problems. Comput Methods Appl Mech Eng 296:73-112

38. Matsubara S, Nishi S, Terada K (2017) On the treatment of heterogeneities and periodic boundary conditions for isogeometric homogenization analysis. Int J Numer Methods Eng 109:15231548

39. Temizer I (2014) Computational homogenization of soft matter friction: isogeometric framework and elastic boundary layers. Int J Numer Methods Eng 100:953-981

40. Fillep S, Mergheim J, Steinmann P (2013) Computational modeling and homogenization of technical textiles. Eng Struct 50:68-73

41. Fillep S, Mergheim J, Steinmann P (2016) Toward an efficient twoscale approach to model technical textiles. Comput Mech 59:385401

42. Espadas-Escalante JJ, van Dijk NP, Isaksson P (2017) A study on the influence of boundary conditions in computational homogenization of periodic structures with application to woven composites. Compos Struct 160:529-537

43. Durville D (2008) A finite element approach of the behaviour of woven materials at microscopic scale. Mechanics of microstructured solids. Springer, Berlin, pp 39-46

44. Durville D (2010) Simulation of the mechanical behaviour of woven fabrics at the scale of fibers. Int J Mater Form 3:1241-1252

45. Nguyen-Thanh N, Zhou K, Zhuang X, Areias P, Nguyen-Xuan H, Bazilevs Y, Rabczuk T (2017) Isogeometric analysis of largedeformation thin shells using RHT-splines for multiple-patch coupling. Comput Methods Appl Mech Eng 316:1157-1178

46. Temizer I, Hesch C (2016) Hierarchical NURBS in frictionless contact. Comput Methods Appl Mech Eng 299:161-186

47. Hesch C, Franke M, Dittmann M, Temizer I (2016) Hierarchical NURBS and a higher-order phase-field approach to fracture for finite-deformation contact problems. Comput Methods Appl Mech Eng 301:242-258

48. Launay J, Hivet G, Duong AV, Boisse P (2008) Experimental analysis of the influence of tensions on in plane shear behaviour of woven composite reinforcements. Compos Sci Technol 68:506-515

49. Tapie E, Tan ESL, Guo YB, Shim VPW (2017) Effects of pretension and impact angle on penetration resistance of woven fabric. Int J Impact Eng 106:171-190

50. Wriggers Computational Contact (2006) Mechanics. Springer, Berlin

51. Betsch P, Grunttmann F, Stein E (1996) A 4-node finite shell element for the implementation of general hyperelastic 3D-elasticity at finite strains. Comput Methods Appl Mech Eng 130:57-79

52. Matsuda T, Nimiya Y, Ohno N, Tokuda M (2007) Elasticviscoplastic behavior of plain-woven GFRP laminates: homog- 
enization using a reduced domain of analysis. Compos Struct 79:493-500

53. Bonet J, Burton AJ (1998) A simple orthotropic, transverly isotropic hyperelastic constitutive equation for large strain computations. Comput Methods Appl Mech Eng 162:151-164
54. Sato M, Muramatsu M, Matsubara S, Nishi S, Terada K, Yashiro K, Kawada T (2017) Numerical plate testing for non-linear multiscale analysis of plate-shaped device. J Jpn Soc Civ Eng Ser A2 (Appl Mech (AM)) 73(2):I_283-I_294

Publisher's Note Springer Nature remains neutral with regard to jurisdictional claims in published maps and institutional affiliations. 